\title{
Optimal Transpiration Boundary Control for Aeroacoustics *
}

\author{
S. Scott Collis, ${ }^{\dagger}$ Kaveh Ghayour ${ }^{\ddagger}$ \\ and Matthias Heinkenschloss ${ }^{\S}$ \\ Rice University \\ Houston, Texas 77005-1892
}

\begin{abstract}
We consider the optimal boundary control of aeroacoustic noise governed by the two-dimensional unsteady compressible Euler equations. The control is the time and space varying wall-normal velocity on a subset of an otherwise solid wall. The objective functional to be minimized is a measure of acoustic amplitude. Optimal transpiration boundary control of aeroacoustic noise introduces challenges beyond those encountered in direct aeroacoustic simulations or in many other optimization problems governed by compressible Euler equations. One nontrivial issue that arises in our optimal control problem is the formulation and implementation of transpiration boundary conditions. Since we allow suction and blowing on the boundary, portions of the boundary may change from inflow to outflow, or vice versa, and different numbers of boundary conditions must be imposed at inflow versus outflow boundaries. Another important issue is the derivation of adjoint equations which are required to compute the gradient of the objective function with respect to the control. Among other things, this is influenced by the choice of boundary conditions for the compressible Euler equations. This paper describes our approaches to meet these challenges and presents results for three model problems. These problems are designed to validate our transpiration boundary conditions and their implementation, study the accuracy of gradient computations, and assess the performance of the computed controls.
\end{abstract}

\section{Nomenclature}

$\mathbf{x}=\left[x_{1}, x_{2}\right]^{T} \quad$ position vector

$\mathbf{v}=\left[v_{1}, v_{2}\right]^{T} \quad$ velocity vector

\footnotetext{
*Preliminary version presented as Paper 2002-2757 at the AIAA 3rd Theoretical Fluid Mechanics Meeting, June 24-27, 2002

${ }^{\dagger}$ Assistant Professor, Department of Mechanical Engineering and Materials Science, collis@ rice.edu, member AIAA.

${ }^{\ddagger}$ Postdoctoral Associate, Department of Computational and Applied Mathematics and Department of Mechanical Engineering and Materials Science, kghayour@caam.rice.edu

${ }_{\S}^{\S}$ Associate Professor, Department of Computational and Applied Mathematics, heinken@rice.edu
} 


\begin{tabular}{|c|c|}
\hline $\mathbf{q}$ & conserved variables $\left[\rho, \rho v_{1}, \rho v_{2}, \rho E\right]^{T}$ \\
\hline $\mathbf{u}$ & primitive variables $\left[\rho, v_{1}, v_{2}, T\right]^{T}$ \\
\hline$\left(t_{0}, t_{f}\right)$ & time period of interest \\
\hline $\mathbf{u}_{0}$ & initial condition at $t_{0}$ \\
\hline $\mathbf{F}^{i}$ & convective flux in $x_{i}$ direction \\
\hline $\mathbf{A}_{i}$ & Jacobian of $\mathbf{F}^{i}$ w.r.t $\mathbf{u}$ \\
\hline$\Omega$ & spatial domain \\
\hline$\Omega_{\mathrm{obs}}$ & observation region \\
\hline$\chi_{\Omega_{\mathrm{obs}}}$ & indicator function \\
\hline$\Gamma$ & boundary of $\Omega$ \\
\hline$\Gamma_{c}$ & subset of $\Gamma$ with control actuation \\
\hline$J$ & objective function \\
\hline$J_{\text {obs }}$ & observation term \\
\hline$J_{\text {reg }}$ & regularization term \\
\hline$\alpha_{i}$ & positive weighting parameters \\
\hline B & vector of BC's \\
\hline$g$ & transpiration control on $\Gamma_{c}$ \\
\hline $\mathcal{G}_{a d}$ & set of admissible controls \\
\hline$\rho$ & density \\
\hline$T$ & temperature \\
\hline$p$ & pressure \\
\hline$p_{a}$ & steady mean-flow pressure \\
\hline$c$ & speed of sound \\
\hline$S$ & entropy \\
\hline$h$ & enthalpy \\
\hline$H$ & stagnation enthalpy \\
\hline$\gamma$ & ratio of specific heats \\
\hline M & Mach number \\
\hline$E$ & total energy per unit mass \\
\hline$\omega$ & vorticity \\
\hline$\lambda$ & adjoint variables \\
\hline$\lambda^{b}$ & adjoint variables associated with BC's \\
\hline$\lambda^{0}$ & adjoint variables associated with IC's \\
\hline$\Psi$ & adjoint characteristic variables \\
\hline$\mu$ & adjoint momentum $\left[\lambda_{2}, \lambda_{3}\right]^{T}$ \\
\hline $\mathbf{n}$ & unit outward normal \\
\hline
\end{tabular}




\section{Introduction}

The goals of this paper are the description of issues arising in the computation of optimal transpiration boundary controls for the minimization of aeroacoustic noise, the introduction of our approaches to deal with these issues, and the presentation of numerical results that support the effectiveness of our approach.

The control objective for aeroacoustic applications usually targets acoustic waves that are typically several orders of magnitude smaller than flow quantities associated with the energetically dominant dynamics. Like the direct simulation of aeroacoustic phenomena, optimal control of aeroacoustics requires proper resolution of small amplitude acoustic fluctuations. However, optimal control imposes additional demands. First, optimal control of aeroacoustic noise requires that dynamic control actuations are properly translated into small amplitude acoustic fluctuations. This can add significant challenges to the simulation. Secondly, to apply gradient based optimization algorithms for the computation of an optimal control, one needs to compute sensitivity information. In our case, where controls are temporally and spatially distributed, this is accomplished using the adjoint equation approach. The implementation of the adjoint equation needs to accurately relate undesired small amplitude acoustic fluctuations to modifications of the control that can reduce them.

Acoustic waves are non-dissipative and non-dispersive; their resolution requires high-order accurate numerical schemes with minimal dissipation and dispersion error. In our state computations, spatial derivative operators are discretized with sixth-order central finite-differences and a fourth-order accurate explicit Runge-Kutta scheme is used to advance the solution in time. We use sponge regions near the far field boundaries to allow acoustic waves and spurious numerical waves to leave the domain without significant reflections. A nonstandard and nontrivial task that arises in our optimal control problem is the formulation and implementation of transpiration boundary conditions. Since we allow suction and blowing on the boundary, portions of the boundary are allowed to change dynamically from inflow to outflow, or vice versa. Different numbers of boundary conditions have to be imposed depending on whether a boundary portion is an inflow or an outflow boundary. Specifically, for subsonic suction only one physical boundary condition is required while three physical boundary conditions are needed for subsonic blowing. Our choice of boundary conditions as well as their implementation in the context of our high-order finite-difference based code is discussed below. Our implementation of the boundary conditions borrows heavily from Sesterhenn. ${ }^{1}$ 
Since the number of control variables in our problem is large, we apply a gradient based algorithm for the computation of an optimal control. The gradient of the objective function with respect to the control is computed using the adjoint equation approach. The general framework underlying our approach in this paper is the same as that used in our previous work ${ }^{2,3}$ and it is similar to many adjoint equation based optimization procedures used for optimization of unsteady problems, although it carefully addresses some subtle but important issues that are sometimes overlooked in other works. In our prior work ${ }^{2}$ on the optimal boundary control of unsteady, two-dimensional viscous compressible flows, we used the discretize-then-optimize approach for gradient computation. In this approach the state equation and objective function are first discretized and the adjoint calculus is applied to the discrete problem, possibly aided by automatic differentiation. While this method generates exact gradient information for the discretized problem, it does so without providing direct physical and mathematical insight into the adjoint partial differential equations (PDEs), especially their boundary conditions, associated with the continuous problem. The latter are important to assess the well-posedness of the optimal control problem and the quality of the computed discretized control as an approximation to the true optimal control. Accurate state discretizations are not sufficient to ensure an accurate discretization of the optimal control problem. For the latter it is also necessary to adequately resolve the adjoint partial differential equations. Gradient computations using the optimize-then-discretize approach, in which one first determines the adjoint partial differential equations and their boundary conditions and then discretizes these, in combination with the aforementioned discretize-then-optimize approach can provide some insight into the issues of well-posedness and approximation quality. We present comparisons of gradient information computed using our optimize-then-discretize approach with finite difference gradient information, which corresponds to discretize-then-optimize approach.

In order to test our approach, three optimal control problems are solved and discussed in this paper. The first test problem shows that the computed optimal controls can produce well-resolved planar acoustic waves of very small amplitude to minimize the observed sound amplitudes by means of wave-cancellation. In the second test problem, the optimal control targets the nopenetration solid wall boundary condition which amplifies the noise amplitude by reflecting the incident waves back into the observation region. The computed optimal control lets the incident acoustic waves pass through the wall, mimicking a non-reflecting boundary condition. The third and last test problem compares the non-reflecting behavior of the optimal transpiration control against two widely used nonreflecting farfield boundary conditions. 


\section{Problem Formulation}

\section{State equations}

The vector $\mathbf{u}=\left(\rho, v_{1}, v_{2}, T\right)^{T}$ denotes the primitive variables and $\mathbf{q}(\mathbf{u})=\left(\rho, \rho v_{1}, \rho v_{2}, \rho E\right)^{T}$ denotes the conserved variables. Here $\rho, v_{i}$ and $T$ are the density, the velocity component in $x_{i}$ direction, and the temperature, respectively. The pressure $p$ and total energy per unit mass $E$ are given by

$$
p=\frac{\rho T}{\gamma \mathrm{M}^{2}} \quad E=\frac{T}{\gamma(\gamma-1) \mathrm{M}^{2}}+\frac{1}{2} \mathbf{v}^{T} \mathbf{v}
$$

where $\mathrm{M}$ is the reference Mach number, $\mathbf{v}$ is the velocity vector, and $\gamma$ is the ratio of specific heats. The convective flux in the $x_{i}$ direction is

$$
\mathbf{F}^{i}(\mathbf{u})=\left(\begin{array}{c}
\rho v_{i} \\
p \delta_{i 1}+\rho v_{1} v_{i} \\
p \delta_{i 2}+\rho v_{2} v_{i} \\
(p+\rho E) v_{i}
\end{array}\right)
$$

where $\delta_{i j}$ is the Kronecker symbol.

The spatial domain occupied by the fluid is $\Omega \subset \mathbb{R}^{2}$, the portion of the solid boundary on which control is exercised is $\Gamma_{c}$, and $\left(t_{0}, t_{f}\right)$ is the time period of interest. By $\mathbf{n}=\left(n_{1}, n_{2}\right)^{T}$ we denote the unit outward normal to the boundary $\Gamma$ of $\Omega$. In our model problems $\Omega=\left\{\mathrm{x} \in \mathbb{R}^{2}: x_{2}>0\right\}$, $\Gamma_{c}=\left\{\mathbf{x} \in \mathbb{R}^{2}: x_{2}=0, a \leq x_{1} \leq b\right\}$ and $\mathbf{n}=(0,-1)^{T}$. The Euler equations can be written in conservative form as

$$
\mathbf{q}(\mathbf{u})_{t}+\sum_{i=1}^{2} \mathbf{F}^{i}(\mathbf{u})_{x_{i}}=\mathbf{0} \quad \text { in }\left(t_{0}, t_{f}\right) \times \Omega
$$

with boundary conditions

$$
\mathbf{B}(\mathbf{u}, \nabla \mathbf{u}, g)=\mathbf{0} \quad \text { on }\left(t_{0}, t_{f}\right) \times \Gamma,
$$

and initial conditions

$$
\mathbf{u}\left(t_{0}, \mathbf{x}\right)=\mathbf{u}_{0}(\mathbf{x}) \quad \text { in } \Omega
$$

The function $g$ in the boundary conditions ( $3 b$ ) is the transpiration boundary control $g=\mathbf{v}^{T} \mathbf{n}$ which is to be determined on the controlled boundary $\Gamma_{c}$. Positive $g$ denotes suction while negative $g$ corresponds to injecting fluid into the domain $\Omega$. The precise form of the boundary conditions 
(3b) will be specified in the following section.

\section{Wall boundary conditions}

Transpiration boundary control $g$ takes place in the near field region where nonlinearities, unsteadiness, and spatial gradients are often significant. Not surprisingly, implementation of a boundary treatment that can accommodate this control mechanism is not an easy task. Moreover, the hyperbolic nature of the Euler equations (and its discretization) often leads to the propagation of nonphysical error waves associated with inappropriately imposed and/or implemented boundary conditions. This can ultimately contaminate the solution everywhere in the domain. This difficulty is particularly acute for high-order central difference discretizations when mated with explicit time-advancement schemes since the discrete dispersion relation permits highly oscillatory numerical error waves that can propagate faster than the physical waves of the system often leading to restrictive time-step constraints.

The implementation of slip-wall boundary conditions (zero normal velocity) for aeroacoustic computations has received attention, most notably the ghost cell method of Tam and Dong ${ }^{4}$ and Kurbatski and Tam. ${ }^{5}$ But, implementation of transpiration type boundary conditions for inviscid compressible flows has received relatively less attention. We view the transpiration boundary condition as an inflow/outflow boundary condition where the number of physical boundary conditions is dictated by the characteristic speeds associated with the unit outward normal $\mathbf{n}$. These speeds are $\mathbf{v}^{T} \mathbf{n}$ (with a multiplicity corresponding to the space dimension) and $\mathbf{v}^{T} \mathbf{n} \pm c$ where $\mathbf{v}$ and $c$ are the local velocity and sound-speed, respectively. When one of these characteristic speeds is negative, the associated characteristic quantity propagates from outside the boundary towards the interior of the domain and a physical boundary condition must be imposed. On the other hand, a positive speed indicates flow of information from the inner domain towards the boundary necessitating the imposition of a numerical boundary condition. Therefore, one physical and three numerical boundary conditions must be imposed for subsonic suction and three physical and one numerical boundary conditions are needed for subsonic blowing. Hence, for an impermeable wall or suction, $\mathbf{v}^{T} \mathbf{n} \geq 0$, and the physical boundary condition

$$
\mathbf{v}^{T} \mathbf{n}-g=0
$$

must be satisfied. However, when fluid is injected into the domain, not only must the wall normal velocity be specified, but, as suggested by the characteristic speeds normal to the boundary, two other flow quantities must be imposed. The appropriate selection of the additional flow quantities that should be constrained can impact both the implementation as well as the accuracy of the final method. Based on extensive experimentation, we have selected entropy and vorticity as the additional flow quantities that are specified in our transpiration boundary condition. This was motivated 
by the well-known fact that entropy and vorticity information propagates along the incoming characteristics and, as shown below, we were able to implement these boundary conditions within our high-order finite difference discretization in such a way that high-accuracy and numerical stability were retained. In summary, for $\mathbf{v}^{T} \mathbf{n}<0$ the boundary conditions

$$
\mathbf{v}^{T} \mathbf{n}=g, \quad \ln \left(\frac{T^{1 /(\gamma-1)}}{\rho}\right)=S_{0}, \quad \partial_{x_{2}} v_{1}-\partial_{x_{1}} v_{2}=\omega_{0}
$$

are imposed. In this study, we require the injected fluid to be isentropic and irrotational by setting $\omega_{0}=S_{0}=0$. Injection of rotational fluid will be explored in future work.

We may combine (4) and (5) into

$$
\mathbf{B}(\mathbf{u}, \nabla \mathbf{u}, g) \stackrel{\text { def }}{=}\left(\begin{array}{c}
\mathbf{v}^{T} \mathbf{n}-g \\
\left(\ln \left(\frac{T^{1 /(\gamma-1)}}{\rho}\right)-S_{0}\right) \min \{g, 0\}^{2} \\
\left(\partial_{x_{2}} v_{1}-\partial_{x_{1}} v_{2}-\omega_{0}\right) \min \{g, 0\}^{2}
\end{array}\right)=\mathbf{0}
$$

Although we have used these boundary conditions successfully in several optimal control applications, mathematical well-posedness of the compressible Euler equations with these boundary conditions has not been established and success of these boundary conditions may be dependent on their implementation, which is discussed below.

\section{Optimal control problem}

In this paper, we solve

$$
\min _{g \in \mathcal{G}_{a d}} J(g)
$$

where the objective function is defined as

$$
\begin{aligned}
J(g) & =J_{\mathrm{obs}}(g)+J_{\mathrm{reg}}(g) \\
J_{\mathrm{obs}}(g) & =\frac{1}{2} \int_{t_{0}}^{t_{f}} \int_{\Omega_{\mathrm{obs}}} \alpha_{0}\left(p-p_{a}\right)^{2} d \mathbf{x} d t \\
J_{\mathrm{reg}}(g) & =\frac{1}{2} \int_{t_{0}}^{t_{f}} \int_{\Gamma_{c}}\left(\alpha_{1} g_{t}^{2}+\alpha_{2} g^{2}+\alpha_{3}(\nabla g)^{2}+\alpha_{4}(\Delta g)^{2}\right) d \Gamma d t
\end{aligned}
$$

In (7) $\alpha_{0}, \ldots, \alpha_{4}$ are positive weighting parameters. The first term is the square of the acoustic amplitude integrated over the observation region $\Omega_{\text {obs }}$ and time horizon $\left[t_{0}, t_{f}\right]$ where $p_{a}$ is the ambient or the steady mean-flow pressure distribution. The term $J_{\text {reg }}(g)$ is a regularization term that enforces certain smoothness requirements on the controls. See Ref. 6 and Ref. 2 for more details. The set $\mathcal{G}_{a d}$ of admissible controls enforces the conditions $g(t, \mathbf{x})=\nabla g(t, \mathbf{x})=0$ for $t \in\left(t_{0}, t_{f}\right)$ and $\mathbf{x} \in \partial \Gamma_{c}$ as well as $g\left(t_{0}, \mathbf{x}\right)=0, \mathbf{x} \in \Gamma_{c}$. The first two conditions ensure that the 
control goes smoothly to zero at the endpoints of the controlled boundary, i.e, at $\partial \Gamma_{c}$. The third condition enforces compatibility between the initial velocity field and boundary data.

\section{Adjoint equations}

We use a gradient-based optimization procedure to solve the optimal control problem. The gradient is computed with the adjoint method. This approach is widely used and the Refs. 2, 3,7-13 represent a small sample of the work on adjoint methods for the optimization of unsteady flows and Refs. 14-18 are few of the many papers discussing adjoint equations for the optimization of steady Euler equations, mostly in the context of optimal design. However, our work here differs in two aspects from the cited literature. First the governing flow equations, especially the boundary conditions are different. Therefore our adjoint PDEs, in particular, our adjoint boundary conditions are different. Secondly, to gain more insight into the mathematical formulation of our problem, we use the optimize-then-discretize approach. This means that we derive the adjoint equations on the PDE level and then discretize our adjoint PDEs to obtain gradient information for the optimization algorithms. The gradient information obtained from this procedure is different than the one obtained by applying the adjoint equation procedure to the discretized version of the optimal control problem (3), (6). While the two outlined procedures do result in different gradient information, one expects or at least hopes that the error between the gradients computed by both approaches goes to zero if the discretization is refined. Of course, for this to happen the optimal control problem and the adjoint PDEs must be formulated properly and the discretization of the optimal control problem as well as of the adjoint PDEs must be suitable for the optimal control context. For our problem and even much simpler optimal problems these are non-trivial issues. Using the optimizethen-discretize approach and the gradient checks, reported on in the section 'Numerical Results,' indicate that our approach is sensible.

In this paper we focus on the aspects of the adjoint equation method that are different in our case from the approaches in the literature. The adjoint equations including boundary conditions are summarized below. Details on their derivation as well as the computation of the objective function gradient may be found in the appendix. Our discretization of the optimal control problem as well as the adjoint equations is discussed in the next section.

The adjoint variables $\boldsymbol{\lambda}=\left(\lambda_{1}, \lambda_{2}, \lambda_{3}, \lambda_{4}\right)^{T}$ to the flow equations (3a) satisfy the differential equation

$$
\mathbf{M}^{T} \boldsymbol{\lambda}_{t}+\sum_{i} \mathbf{A}^{i^{T}} \boldsymbol{\lambda}_{x_{i}}=\mathbf{r}
$$

where $\mathbf{M}=\mathbf{q}_{\mathbf{u}}(\mathbf{u})$ and $\mathbf{A}^{i}=\mathbf{F}_{\mathbf{u}}^{i}(\mathbf{u})$. The source term $\mathbf{r}$ in (8) is obtained by differentiating the 
right hand side in (7b) with respect to the primitive variables and is given by

$$
\mathbf{r}=\alpha_{0} \frac{p-p_{a}}{\gamma \mathrm{M}^{2}}\left(\begin{array}{c}
T \\
0 \\
0 \\
\rho
\end{array}\right) \chi_{\Omega_{\mathrm{obs}}}
$$

where $\chi_{\Omega_{\mathrm{obs}}}$ is the indicator function, i.e., $\chi_{\Omega_{\mathrm{obs}}}(\mathbf{x})=1$ for $\mathbf{x} \in \Omega_{\mathrm{obs}}$ and $\chi_{\Omega_{\mathrm{obs}}}(\mathbf{x})=0$ otherwise. The final time condition for the adjoint variables is given by

$$
\boldsymbol{\lambda}\left(t_{f}, \mathbf{x}\right)=0
$$

In the appendix, the adjoint boundary conditions are written in a compact form in terms of the adjoint characteristic variables. In the following, however, the adjoint boundary conditions are stated in terms of the adjoint variables $\boldsymbol{\lambda}$ for sake of completeness. We also define the adjoint momentum as $\boldsymbol{\mu}=\left(\lambda_{2}, \lambda_{3}\right)^{T}$. For suction $\left(g=\mathbf{v}^{T} \mathbf{n} \geq 0\right)$ we obtain the three boundary conditions

$$
\begin{aligned}
g\left(\lambda_{1}+\mathbf{v}^{T} \boldsymbol{\mu}+\frac{\mathbf{v}^{T} \mathbf{v}}{2} \lambda_{4}\right) & =0 \\
\boldsymbol{\mu}^{T} \mathbf{n}+\frac{\gamma}{\gamma-1} g \lambda_{4} & =0 \\
g\left(\boldsymbol{\mu}^{T} \mathbf{s}+\mathbf{v}^{T} \mathbf{s} \lambda_{4}\right) & =0
\end{aligned}
$$

The solid surface is a special case of suction with $g=0$ and it can be seen from (11) that only one boundary condition

$$
\boldsymbol{\mu}^{T} \mathbf{n}=0
$$

is required. For blowing ( $g=\mathbf{v}^{T} \mathbf{n}<0$ ), we obtain two boundary conditions

$$
\begin{aligned}
g\left(\boldsymbol{\mu}^{T} \mathbf{s}+\mathbf{v}^{T} \mathbf{s} \lambda_{4}\right) & =0 \\
\boldsymbol{\mu}^{T} \mathbf{n}+\frac{\gamma}{\gamma-1} g \lambda_{4}+\frac{\mathrm{M}^{2}}{T} g\left(\lambda_{1}+\mathbf{v}^{T} \boldsymbol{\mu}+\frac{\mathbf{v}^{T} \mathbf{v}}{2} \lambda_{4}\right) & =0 .
\end{aligned}
$$

Looking at the characteristics of the adjoint equation (8), one would expect only one boundary condition in the case $g=\mathbf{v}^{T} \mathbf{n}<0$. A full analysis of these adjoint boundary conditions is in progress. However, it should be noted that the derived boundary conditions for suction and blowing are compatible as $g \rightarrow 0$. 


\section{Implementation}

In this section, we focus on the discretization scheme and the implementation of the transpiration boundary condition for the state equations.

\section{Discretization}

Numerical simulation of aeroacoustic phenomena demands high numerical accuracy (i.e. low dissipation and dispersion) to accurately resolve convective flow-features over a wide range of space/time scales and amplitudes. To meet this need, most prior approaches have utilized highorder accurate finite difference methods such as compact schemes ${ }^{19}$ and the dispersion relation preserving methods. ${ }^{20}$ Our study focuses on the application of optimal control to aeroacoustic problems and for this purpose, we have initially chosen to use the sixth-order central finitedifference method with explicit high-wavenumber damping and simple boundary treatments. This combination posses excellent dispersion relation preserving properties (see e.q., Ref. 21) while its simplicity allows us to explore the issues involved in applying optimal control theory to aeroacoustic flows while avoiding complications that may arise due to more complex discretizations.

With this background, our Euler flow solver is based on a conservative extension of the explicit finite-difference method described in Ref. 21. The Euler equations are formulated in a generalized coordinate system where the physical domain is mapped to a computational space; a unit square divided into an equally spaced grid system. This transformation allows clustering of grid points in regions of high gradients, simplifies the implementation of the boundary conditions, and allows the code to be used for moderately complex geometries. While the code supports optimized finite-differences with up to seven point stencils, for this study spatial derivatives are approximated using standard sixth-order accurate central differences in the interior with third-order biased and one-sided differences used at boundaries that are designed to enhance stability when used with explicit time advancement methods. ${ }^{22}$ To suppress the growth of high-wavenumber error modes, a fourth-order artificial dissipation term is added to the right hand side of the discretized equations. This dissipation term is computed using fourth-order accurate finite-differences and the dissipation parameter is chosen to damp out the error modes while avoiding excessive dissipation in the resolved scales as established through numerical experimentation. Sponge terms ${ }^{21,23}$ are used in the vicinity of the farfield boundary with a one-dimensional Riemann invariant treatment at the farfield boundary. ${ }^{21}$

\section{Implementation of boundary conditions}

As discussed earlier, the state boundary conditions (4) and (5) do not provide enough information to populate $\mathbf{u}$ on the boundary. For subsonic suction the physical boundary condition (4) has to be augmented by three numerical boundary conditions and for subsonic blowing one numerical boundary condition is needed in addition to the three physical boundary conditions (5). Analogous 
considerations apply to the adjoint PDE. However, since the adjoint PDEs are solved backward in time, characteristics that are outgoing for the state equation are incoming for the adjoint equations and vice versa. The following describes our implementation of state and adjoint boundary conditions.

\section{Implementation of wall transpiration boundary conditions}

Most wall boundary-condition treatments in the literature focus on solid walls and the numerical implementation of the no-penetration boundary condition. In the course of our study, we tried to modify several existing boundary-condition treatments to accommodate wall-normal suction and blowing. First, we tried the common treatment of solving the continuity equation for density on the boundary, imposing the normal velocity actuation, and extrapolation of the remaining state variables from the inner domain. This treatment produces considerable numerical noise that corrupts the dilatation and vorticity fields and severely reduces the allowable time-step for a stable solution. Next, we tried the one-dimensional characteristic-based approaches of Thompson, ${ }^{24,25}$ Poinsot and Lele, ${ }^{26}$ and Giles. ${ }^{27}$ We could not successfully modify any of these methods to actuate a time and space varying suction and blowing control without eventual blow-up of the simulation.

We eventually arrived at an implementation with acceptable accuracy and stability properties that is based on the approach originally formulated by J. Sesterhenn ${ }^{1}$ who expresses the inviscid part of the equations as a decomposition into several plane waves aligned with the numerical grid in an attempt to merge finite differencing with schemes based on compatibility equations such as Moretti's $\lambda$-scheme. ${ }^{28}$ In this approach, transport equations are written for pressure, normal and tangential velocities in terms of the 'pseudo' acoustic wave amplitudes. These 'pseudo' waves may not have any physical significance in two or three dimensional flows but in one dimension coincide with the temporal change of acoustic wave amplitudes closely related with the Riemann invariants of homentropic flows. Also, the introduction of these pseudo-waves allows a more direct and natural implementation of wall boundary conditions as opposed to the locally one-dimensional inviscid approximation of Poinsot and Lele. ${ }^{26}$ We use Sesterhenn's decomposition only on the wall boundary to implement the transpiration boundary conditions (4) and (5). In the following, body coordinates $\mathbf{s}-\mathbf{n}$ are used, where $\mathbf{n}$ denotes the outward normal direction to the boundary and $\mathbf{s}=\left(s_{1}, s_{2}\right)^{T}=\left(-n_{2}, n_{1}\right)^{T}$ is the unit tangential vector. We define the following 'pseudo-waves' in the tangential and normal directions:

$$
\begin{aligned}
& X_{\mathbf{s}}^{ \pm}=\left(\mathbf{v}^{T} \mathbf{s} \pm c\right)\left(\frac{1}{\rho c} \partial_{\mathbf{s}} p \pm \partial_{\mathbf{s}} \mathbf{v}^{T} \mathbf{s}\right) \\
& X_{\mathbf{n}}^{ \pm}=\left(\mathbf{v}^{T} \mathbf{n} \pm c\right)\left(\frac{1}{\rho c} \partial_{\mathbf{n}} p \pm \partial_{\mathbf{n}} \mathbf{v}^{T} \mathbf{n}\right)
\end{aligned}
$$

where $c$ denotes the speed of sound. Transport equations for the velocity components and pressure 
can be recast in terms of these pseudo-waves as

$$
\begin{aligned}
\partial_{t} \mathbf{v}^{T} \mathbf{n}= & -\frac{1}{2}\left(X_{\mathbf{n}}^{+}-X_{\mathbf{n}}^{-}\right)-\mathbf{v}^{T} \mathbf{s} \partial_{\mathbf{s}} \mathbf{v}^{T} \mathbf{n}-\left(\mathbf{v}^{T} \mathbf{s}\right)^{2} / R, \\
\partial_{t} \mathbf{v}^{T} \mathbf{s}= & -\frac{1}{2}\left(X_{\mathbf{s}}^{+}-X_{\mathbf{s}}^{-}\right)-\mathbf{v}^{T} \mathbf{n} \partial_{\mathbf{n}} \mathbf{v}^{T} \mathbf{s}+\mathbf{v}^{T} \mathbf{s} \mathbf{v}^{T} \mathbf{n} / R, \\
\partial_{t} p= & -\frac{\rho c}{2}\left(X_{\mathbf{s}}^{+}+X_{\mathbf{s}}^{-}+X_{\mathbf{n}}^{+}+X_{\mathbf{n}}^{-}\right)+\rho c^{2} \mathbf{v}^{T} \mathbf{n} / R,
\end{aligned}
$$

where $R$ denotes the radius of curvature. At boundary points, $X_{\mathrm{s}}^{ \pm}$are determined from the boundary data available at the current time. However, in the $\mathbf{n}$ direction, the Mach number $\mathbf{M}_{\mathbf{n}}=\mathbf{v}^{T} \mathbf{n} / c$ determines where the $X_{\mathbf{n}}$ pseudo-waves originate. For example, when the flow is locally subsonic, $X_{\mathbf{n}}^{-}$enters the domain from outside while $X_{\mathbf{n}}^{+}$leaves the domain, as seen in Fig. 1. Waves which enter the domain are specified so that the imposed physical boundary conditions are satisfied. As mentioned earlier, one physical boundary condition, $\mathbf{v}^{T} \mathbf{n}=g$, must be imposed on the boundary for subsonic suction. The unknown pseudo-wave $X_{\mathbf{n}}^{-}$is determined from (15a),

$$
X_{\mathbf{n}}^{-}=X_{\mathbf{n}}^{+}+2\left(\partial_{t} g+\mathbf{v}^{T} \mathbf{s} \partial_{\mathbf{s}} g+\left(\mathbf{v}^{T} \mathbf{s}\right)^{2} / R\right)
$$

The time derivative of pressure can now be computed by substituting for $X_{\mathbf{n}}^{-}$from (16) in (15c). The energy equation can be written in terms of entropy as

$$
\partial_{t} S+\mathbf{v}^{T} \mathbf{s} \partial_{\mathbf{s}} S+\mathbf{v}^{T} \mathbf{n} \partial_{\mathbf{n}} S=0
$$

where $\partial_{n} S$ is computed from the interior domain using a one-sided finite-difference stencil allowing us to compute the time derivative of entropy from (17). Similarly, if $\partial_{\mathbf{n}} \mathbf{v}^{T} \mathbf{s}$ is computed by a one-sided finite-difference stencil, the time derivative of $\mathbf{v}^{T} \mathbf{s}$ can also be computed from (15b). Now, we have $\partial_{t} \mathbf{v}^{T} \mathbf{s}, \partial_{t} \mathbf{v}^{T} \mathbf{n}, \partial_{t} p$, and $\partial_{t} S$ at our disposal and can easily compute the temporal derivative of any other flow quantity. For instance, to compute $\partial_{t} \rho$, one can use the Gibbs equation

$$
d h=T d S+\frac{d p}{\rho}
$$

in conjunction with the equation of state (1) to write the density time derivative in terms of entropy and pressure.

For subsonic blowing, however, three physical boundary conditions must be imposed, $\mathbf{v}^{T} \mathbf{n}=$ $-g, S=0$ and $\omega=0$ where $S$ and $\omega$ denote entropy and vorticity, respectively. Again, the unknown pseudo-wave is determined from (16) and the pressure time derivative is subsequently computed from (15c). The time derivative of density is found by enforcing zero rate of change in entropy in (18)

$$
\partial_{t} \rho=\frac{1}{c^{2}} \partial_{t} p .
$$


Vorticity $\omega$ is given by

$$
\omega=-\partial_{\mathbf{s}} \mathbf{v}^{T} \mathbf{n}+\partial_{\mathbf{n}} \mathbf{v}^{T} \mathbf{s}-\frac{\mathbf{v}^{T} \mathbf{s}}{R}
$$

where $\partial_{\mathbf{s}} \mathbf{v}^{T} \mathbf{n}=\partial_{\mathbf{s}} g$ is a known quantity. The boundary condition $\omega=0$ is enforced 'weakly' by substituting $\partial_{\mathbf{n}} \mathbf{v}^{T} \mathbf{s}=\partial_{\mathbf{s}} \mathbf{v}^{T} \mathbf{n}+\mathbf{v}^{T} \mathbf{s} / R$ in the right hand side of (15b).

\section{Implementation of adjoint boundary conditions}

Our treatment of the adjoint boundary conditions differs from that of the state boundary conditions described above. Although we could have applied a boundary treatment similar to that used for the state, our adjoint boundary condition implementation is instead based on simple extrapolation of appropriate adjoint characteristic quantities similar to that described for the Euler equations in Ref. 29. This simple implementation has been sufficient for all of the adjoint computations presented here. We point out, that the optimize-then-discretize framework allows us to use different

implementations in the state and adjoint discretizations and we have made use of that flexibility here.

There exist nonsingular matrices $\mathbf{P}, \mathbf{L}$ and a diagonal matrix $\boldsymbol{\Lambda}$ with entries $\mathbf{v}^{T} \mathbf{n}, \mathbf{v}^{T} \mathbf{n}, \mathbf{v}^{T} \mathbf{n}+c$, $\mathbf{v}^{T} \mathbf{n}-c$ such that

$$
\sum_{i} n_{i} \mathbf{A}^{i^{T}}=\mathbf{L}^{-T} \mathbf{\Lambda} \mathbf{P}^{T}
$$

(for details see the appendix). Because the adjoint equations are solved backward in time, a negative eigenvalue $\mathbf{v}^{T} \mathbf{n}, \mathbf{v}^{T} \mathbf{n} \pm c$ indicates flow of information from the inner domain towards the boundary. In order to populate the adjoint vector on the boundary, numerical boundary conditions are needed to complement the derived adjoint boundary conditions. In this work, we simply extrapolate the components of $\boldsymbol{\Psi}=\left(\Psi_{1}, \Psi_{2}, \Psi_{3}, \Psi_{4}\right)^{T} \stackrel{\text { def }}{=} \mathbf{P}^{T} \boldsymbol{\lambda}$ that correspond to negative propagation speeds in the direction normal to the boundary.

For subsonic suction, a third order extrapolation of $\Psi_{4}$ from the inner domain provides the numerical boundary condition that complements the adjoint boundary conditions (11) (or their equivalent expression (33)-(35) in terms of the characteristic adjoint variables).

For subsonic blowing, we extrapolate $\Psi_{1}$ and $\Psi_{4}$ while $\Psi_{2}, \Psi_{3}$ are determined from the adjoint boundary conditions (13), more precisely their equivalent expression (35), (39) in terms of the characteristic adjoint variables derived in the appendix.

\section{Farfield sponge treatment for the state and adjoint equations}

We use sponges at the farfield boundaries to prevent outgoing acoustic and numerical waves from being reflected in the domain. A sponge is applied by adding a source term of the form $-\theta(\mathbf{x})(\mathbf{q}-\hat{\mathbf{q}})$ to the Euler equations in the sponge region. The function $\theta(\mathbf{x})$ is a strictly increasing function which is only nonzero in the sponge and varies from zero at the inner edge to a large value 
at the outer edge. The state solution is forced to approach the prescribed field $\hat{\mathbf{q}}$ in the sponge region. The adjoint of the sponge can be easily seen to be of the form $\theta(\mathbf{x}) \mathbf{M}^{T} \boldsymbol{\lambda}$ which is added to the right hand side of (8). This sponge term forces the adjoint variable $\boldsymbol{\lambda}$ to effectively vanish in the sponge region.

\section{Numerical Results}

In our model problems $\Omega=\left\{\mathrm{x} \in \mathbb{R}^{2}: x_{2}>0\right\}, \Gamma_{c}=\left\{\mathrm{x} \in \mathbb{R}^{2}: x_{2}=0, a \leq x_{1} \leq b\right\}$ and $\mathbf{n}=(0,-1)^{T}$.

\section{Test case 1}

In this section we present optimal control results for the test problem depicted in Fig. 2. In the following, the source period $T_{p}$ and the wave length $L$ are used for nondimensionalization. A time harmonic line source is located at a distance $H=5$ from a solid wall. The computational domain is $\Omega=[-3.5,3.5] \times[0,7]$ with periodic boundary conditions in the horizontal direction and a sponge-type non-reflecting far-field boundary condition applied at the top boundary. The control objective is (7) with observation region $\Omega_{\mathrm{obs}}=[-2,2] \times[1 / 2,3 / 2]$ (the rectangular box shown in each of the contour plots of Figs. 3, 5(b)) and time interval is from $t_{0}=30$ to $t_{f}=50$. As the pressure fluctuations are usually very small, the weight $\alpha_{0}$ in the control objective (7) is chosen to be large relative to the other weights. We use $\alpha_{0}=10^{6}, \alpha_{1}=10^{-3}, \alpha_{2}=10^{-7}$, $\alpha_{3}=\alpha_{4}=10^{-3}$. The selection of weights is an important engineering design question. When solving optimal control problems for specific applications, a more systematic way to determine their influence on the controlled flow is desirable. However, for the test cases presented here, we have selected the weights based on our experience in Ref. 2 as well as experimentation in the context of the present test problems.

Wall-normal transpiration constitutes the boundary control, defined over $[-3,3] \times\left[t_{0}, t_{f}\right]$. The time interval is comprised of 800 uniform time-steps of size $\Delta t=0.025$ and the spatial mesh $(141 \times 141)$ has uniform spacing of $\Delta x_{1}=\Delta x_{2}=0.05$. As the line source is harmonic and the effect of nonlinearities away from the source region is negligible, the flow field exhibits a limit-cycle behavior with period $T_{p}$ as $t \rightarrow \infty$. The initial time $t_{0}$ is chosen large enough for this limit-cycle pattern to be effectively established in the domain. Figure 3(a) shows contours of pressure fluctuations about the ambient pressure, $p-p_{\mathrm{a}}$, for the uncontrolled flow within one period of oscillation.

The analytical solution for the infinite dimensional no-control problem can be easily found by superimposing an image line source at $x_{2}=-H$. The second component of velocity, $v_{2}$, and 
pressure, $p$, can be written as

$$
\begin{aligned}
v_{2} & =4 v_{m} \sin \left(2 \pi x_{2}\right) \cos \left(2 \pi\left(H-t+\frac{1}{4}\right)\right), \\
p & =4 p_{m} \cos \left(2 \pi x_{2}\right) \cos (2 \pi(H-t)) .
\end{aligned}
$$

In (21), the velocity and pressure amplitude of the harmonic line source are denoted by $v_{m}$ and $p_{m}$, respectively. Equation (21a) satisfies the inviscid wall boundary condition at $x_{2}=0$ at all times and further examination of (21b) shows that for $t=H / c+(2 n+1) / 4, n=0,1,2,3, \ldots$, the pressure fluctuation vanishes everywhere in the domain. This behavior is seen in the second and fourth plots of Fig. 3(a).

For this test case the value of the objective function (7) at the initial iterate $g_{0}=0$ (no control) is $J\left(g_{0}\right)=J_{\text {obs }}\left(g_{0}\right)=1.2 \times 10^{-3}$. After 15 nonlinear conjugate gradient iterations, ${ }^{2}$ the computed control $g_{15}$ reduces the observation term in the objective function to $J_{\text {obs }}\left(g_{15}\right)=6.6 \times 10^{-4}$ which is about $45 \%$ of its initial value. Because of our choice of weights $\alpha_{i}$, we obtain that $J_{\text {reg }}\left(g_{15}\right) \approx$ $10^{-10} J_{\text {obs }}\left(g_{15}\right)$, i.e., the value $J\left(g_{15}\right)$ of the objective function (7) can be equated with $J_{\text {obs }}\left(g_{15}\right)$. The same is also true for the other test cases. The acoustic pressure contours for the controlled run are shown in Fig. 3(b). In order to analyze the behavior of the computed control, the time history of the control at $x_{1}=0, \pm 1 / 4$ is plotted in Fig. 4. Figure 4(a) depicts the time history of control at $x_{1}=0$. It can be seen that the control has no difficulty in picking up the source frequency and the control oscillates with an approximately constant amplitude for most of the time window $\left(t_{0}, t_{f}\right)$. Close to the final time, the control loses the harmonic behavior and becomes approximately constant. Figure 4(b) shows the time history of control at three locations on the wall, $x_{1}=0, \pm 1 / 4$, separated by a quarter wave length. This plot demonstrates that the control at these three locations are exactly in phase. Further checks reveal that the control indeed is constant across the control region and is only a function of time. Because the lower edge of the observation region is located at a distance $1 / 2$ above the wall, it takes about $1 / 2$ time units for the effect of the boundary actuation to be felt in the observation region - explaining the behavior of the control near the final time shown in Fig. 5(a). From $t=49.5$ onward, the control cannot affect the observation term and, hence, it tries to minimize the contribution of the regularization term. The contribution of the spatial derivatives $g_{x_{1}}$ and $g_{x_{1} x_{1}}$ to (7) is zero and the contribution of the time regularization term $\left\|g_{t}\right\|_{2}^{2}$ is approximately $4 \pi^{2} \approx 39.5$ times larger than the regularization term $\|g\|_{2}^{2}$ for a simple harmonic oscillation. Therefore, the main focus of the optimal control in the time period $\left(39.5, t_{f}\right)$ is to initially reduce $g_{t}$, which is clearly achieved by creating a plateau near the final time $t_{f}$. To understand the effect of the computed control, one can subtract the uncontrolled pressure field from the optimal control pressure field as shown in Fig. 5(b). As the amplitude of flow quantities are small, the nonlinear terms in the governing equations are negligible and 
the contour plot of Fig. 5(b) isolates the effect of boundary actuation. It is clearly seen that the boundary control creates a nearly planar wave to counter the wave system of (21) in the observation region. A perfect cancellation is not possible because the wave produced by the control cannot cancel the wave system of (21) at all times. The optimal control targets instants of time at which the observation region has high amplitude waves (the first and third snapshots of Fig. 3(a) and tries

to reduce these waves by producing the wave shown in Fig. 5(b). The control slightly disturbs the approximately silent instants observed in the second and fourth plots of Fig. 3(b).

\section{Test case 2}

In this test case, we control the scattered and refracted wave pattern arising from the interaction of a monopole sound source with an inviscid vortex. Again, all flow quantities are nondimensionalized with source period $T_{p}$ and acoustic wavelength $L$. The monopole sound source, modeled as a source term in the energy equation, is located at $(0,5)$ and interacts with an inviscid vortex ${ }^{30}$ of circulation $2 \pi / 5$ (counter-clockwise) and radius $1 / 2$ located at $(0,3.5)$. The computational domain, control objective, and the spatial and temporal discretizations are identical to that of test case 1. Sponge-type nonreflecting far-field boundary conditions are now used on the left, right and top boundaries. The distance between the vortex and the solid wall is large enough to comfortably ignore the effect of the image vortex in the optimization time interval. Hence, the vortex is considered to be stationary and the mean flow pressure distribution to be steady. The time interval is comprised of 600 uniform time-steps of size $\Delta t=0.025$ from time $t_{0}=30$ to time $t_{f}=45$. Again, the initial time is larger than the time required for the limit-cycle pattern to be effectively established in the domain.

After 20 optimization iterations the control objective is reduced from $J_{\text {obs }}\left(g_{0}\right)=0.49$ to $J_{\text {obs }}\left(g_{20}\right)=0.34$. The acoustic pressure contours for the no control and optimal control simulations are shown in Fig. 6(a)-(b) respectively. In the no control simulation, the scattered wave behind the vortex is mostly observed in the right half of the observation region, which is due to the counter-clockwise circulation of the vortex, and the left half is relatively silent. The solid wall intensifies the incident waves and forces the waves to move horizontally.

However, focusing on the observation region of the optimal control run reveals that the main difference between the two runs is the slanted contours of the optimal control simulation. In order to understand the control mechanism, another simulation is performed where the solid wall is replaced with a nonreflecting boundary condition based on Riemann extrapolation. Figure 6(c) shows four snapshots of the acoustic pressure field at the same instants in time using the Reimann boundary treatment. The pressure contours in the observation region for this run are very similar to that of the optimal control run, both slanting at an angle to the horizon unlike the almost flat contours of the uncontrolled run. This suggests that the optimal control attempts to mimic 
an absorbing boundary. The computed optimal control makes the wall nearly transparent to the incident waves, thereby preventing them from reflecting and intensifying the sound measured in the observation region.

In the no-control run, the refracted/reflected wave pattern seen in the middle of the observation region is approximately horizontal. But, the wave system produced by the boundary actuation (Fig. 7(b)) and the wave pattern observed in the center of the optimal control plots (Fig. 6) slant in opposite directions. The production of the slanted wave of Fig. 7(b) by the boundary actuation is further validated by Fig. 7(a) where the time history of control at three positions, $x_{1}=-0.25,0,0.25$, separated by a quarter acoustic wavelength, is plotted. The phase difference in control actuation at these three locations allows for the production of waves with slanted fronts. It is interesting to note that the control amplitude is smaller at $x_{1}=-0.25$ than the other two locations. This shows that the optimal control avoids disturbing the relatively silent left half of the observation region while attenuating the noisy right half of this region.

\section{Test case 3}

In test case 2 , we argued that the computed transpiration boundary actuation effectively rendered the controlled surface transparent to incident waves. Test case 3 allows us to quantify how closely a transpiration wall is capable of mimicking a nonreflecting boundary condition. The computational domain is $\Omega=[-12,12] \times[0,14]$ with periodic boundary conditions enforced in the horizontal direction and a sponge nonreflecting boundary treatment in the vicinity of the top boundary. The control objective is identical in form to that of the previous test cases and is defined over $\Omega_{\mathrm{obs}}=[-5,5] \times[5,9]$ and time horizon $[2,9]$ comprised of 175 uniform time-steps $\Delta t=0.04$. The spatial mesh $(241 \times 141)$ has uniform spacing $\Delta x_{1}=\Delta x_{2}=0.1$ in both directions and transpiration control is allowed over the entire bottom boundary. The initial condition is a Gaussian acoustic pulse of amplitude $\epsilon=10^{-3}$ with standard deviation $\sigma=0.25$, centered at mean height $x_{2}^{*}=8$ above the wall,

$$
\begin{aligned}
v_{1} & =0, \\
v_{2} & =-\frac{\epsilon}{2} e^{-\frac{1}{2}\left(\frac{x_{2}-x_{2}^{*}}{\sigma}\right)^{2}}, \\
p-p_{a} & =-\rho_{a} c_{a} v_{2}, \\
\rho-\rho_{a} & =\frac{p-p_{a}}{c_{a}^{2}} .
\end{aligned}
$$

In (22), the subscript ' $a$ ' denotes the ambient condition assumed to be a uniform quiescent flow where $\rho_{a}=T_{a}=1$ and $c_{a}=2$. The acoustic pulse propagates at the ambient speed of sound $c_{a}=2$ towards the wall and at $t_{0}$, the beginning of the optimization horizon, it is located at $x_{2}=4$. For the no control simulation, the pulse reflects off the solid wall, passes through $\Omega_{\mathrm{obs}}$, 
and reaches $x_{2}=10$ at the final time $t_{f}$. We use $\alpha_{0}=10^{6}, \alpha_{1}=10^{-3}, \alpha_{2}=10^{-4}, \alpha_{3}=\alpha_{4}=$ $10^{-3}$. Optimization starts at the no-control configuration with $J\left(g_{0}\right)=J_{\text {obs }}\left(g_{0}\right)=9.19 \times 10^{-1}$, $\left\|\nabla J\left(g_{0}\right)\right\|_{\mathcal{G}}=3430$ and is terminated after 133 nonlinear conjugate gradient iterations $J_{\text {obs }}\left(g_{133}\right)=$ $3.98 \times 10^{-5},\left\|\nabla J\left(g_{133}\right)\right\|_{\mathcal{G}}=2.59$. The norm, $\|g\|_{\mathcal{G}}$, which is specified in Ref 2 , is related to $\int_{t_{0}}^{t_{f}} \int_{\Gamma_{c}} g^{2}+g_{t}^{2}+|\nabla g|^{2}+|\Delta g|^{2} d \Gamma d t$, and is not the Euclidean norm. Four snapshots of the acoustic pressure contours of the no control and optimally controlled flow are shown in Fig 8. We note that while the uncontrolled flow is one-dimensional, the controlled flow is two-dimensional due to the finite sized control region on the bottom wall.

Fig. 8(a) shows that the observation region is quiet except for the time interval that the reflection off the solid wall passes through it. Therefore, the optimal control actuation cannot decrease the objective function unless it eliminates the reflection from the wall and makes the controlled wall transparent to the incident acoustic pulse. This behavior is evident in Figure 8(b) where the control actuation has allowed the central portion of the acoustic pulse between $[-5,5]$ to pass through the wall without noticeable reflection.

In order to access the performance of the optimal control transpiration $\mathrm{BC}$ in producing an essentially nonreflecting boundary condition, we remove the lower wall and enforce the well known nonreflecting treatments: 1) Riemann extrapolation and 2) sponge. Then, we compute $J_{\text {obs }}=\frac{1}{2} \int_{t_{0}}^{t_{f}} \int_{\Omega_{\mathrm{obs}}} \alpha_{0}\left(p-p_{a}\right)^{2} d \mathbf{x} d t, \alpha_{0}=10^{6}$, for each case to estimate the amount of reflections produced by each boundary treatment. For the optimal transpiration boundary control we find $J_{\mathrm{obs}} \approx 4 \times 10^{-5}$, Riemann extrapolation boundary conditions give $J_{\mathrm{obs}} \approx 6.4 \times 10^{-5}$, and sponge boundary conditions lead to $J_{\mathrm{obs}} \approx 1 . \times 10^{-6}$. We note that the Riemann condition performs somewhat worse than the sponge since Riemann treatments are well known to reflect the non-smooth, numerical error waves. The amount of reflections in the observation region for the controlled wall is less than the Riemann treatment while it is considerably higher than the sponge treatment. As the transpiration and Riemann boundary condition implementations are both based on the flow characteristics, the Riemann $\mathrm{BC}$ is a reasonable reference against which the performance of the transpiration BC can be measured. The fact that the optimal control boundary performs slight better that the Reimann $\mathrm{BC}$ is likely due to the numerical error associated with extrapolation in the Reimann BC. Figure 9 compares the amplitudes of the reflected waves by plotting the time history of pressure fluctuations in the center of the observation region. The maximum sound amplitude for the optimal control simulation is slightly less than half of the peak reflection amplitude due to the Riemann treatment, while the peak reflection amplitude of the sponge treatment is about an order of magnitude smaller than the other two. 


\section{Gradient accuracy}

As we have mentioned earlier, we use the optimize-then-discretize approach to compute gradient information, i.e., we first derive adjoint equations and the objective function gradient on the PDE level and then discretize the resulting expressions. We denote the result by $(\nabla J(g))_{h}$ to indicate that discretization is performed after differentiation. The resulting derivative approximation differs from gradient $\nabla\left(J_{h}(g)\right)$ obtained by first discretizing the optimal control problem and then computing the gradient of the resulting finite dimensional objective function $J_{h}(g)$. If adjoint computations are performed correctly and if discretizations of state equation, objective function and adjoint equations are chosen properly, one would expect that $(\nabla J(g))_{h}-\nabla\left(J_{h}(g)\right) \rightarrow 0$ as the discretization level $h$ is refined. Moreover, the rate with which $(\nabla J(g))_{h}-\nabla\left(J_{h}(g)\right)$ goes to zero should be related to the approximation properties of the underlying discretization schemes. That, in fact, the convergence $(\nabla J(g))_{h}-\nabla\left(J_{h}(g)\right) \rightarrow 0$ as $h \rightarrow 0$ is obtained is not at all straightforward. Even for optimal control problems far less complex than the one considered here, Refs. 31, 32 offer some sobering results.

The results in this section provide evidence that in our treatment $(\nabla J(g))_{h}-\nabla\left(J_{h}(g)\right) \rightarrow 0$ as the discretization level $h$ is refined. Since we do not have access to the gradient of the discretized objective function $J_{h}(g)$, we choose an arbitrary unit direction $\delta g$ in the space of admissible controls and compare $\left\langle(\nabla J(g))_{h}, \delta g\right\rangle_{\mathcal{G}}$ with a finite difference approximation of $\left\langle\nabla\left(J_{h}(g)\right), \delta g\right\rangle_{\mathcal{G}}$. Here $\left\langle g_{1}, g_{2}\right\rangle_{\mathcal{G}}$ is a weighted Euclidian norm corresponding to $\|\cdot\|_{\mathcal{G}}$, which is specified in detail in Ref. 2. To select a suitable finite difference step size $\epsilon$, we evaluate the discretized objective function at $\pm \epsilon \delta g$ and $\pm 2 \epsilon \delta g$ and compare the second order approximation $\left[J_{h}(g+\epsilon \delta g)-J_{h}(g-\right.$ $\epsilon \delta g)] /(2 \epsilon)$ with a fourth order finite difference approximation. The finite difference step size $\epsilon$ is varied until these two approximations agree to a relative error of less than $10^{-6}$ (for test case (3)). For example, for test case (3) using a $241 \times 141$ grid and $g=0$, the computed second order approximation to $\left\langle\nabla\left(J_{h}(g)\right), \delta g\right\rangle_{\mathcal{G}}$ is -535.1712 and the computed fourth order approximation is -535.1714 . The corresponding directional derivative computed using the optimize-then-discretize approach is $\left\langle(\nabla J(g))_{h}, \delta g\right\rangle_{\mathcal{G}}=-535.1872$.

Figure 10 shows the results of grid convergence studies using grids ranging in size from $241 \times$ 141 to $961 \times 561$. For our computations, the solution at the finest discretization $961 \times 561$ is assumed to be the reference solution and is denoted by subscript ' $\mathrm{f}$ '. In Fig. 10, three relative error measures have been plotted against the number of mesh points $N_{x_{1}}$, where $N_{x_{1}}$ is the number of grid points in $x_{1}$ direction and the number of grid points in the $x_{2}$ direction is $(7 / 12)\left(N_{x_{1}}-\right.$ $1)+1$, chosen to give the same grid spacing in both directions. In Fig. 10, $J$ denotes the value of the objective function (7a), DA refers to the approximate directional derivative $\left\langle(\nabla J(g))_{h}, \delta g\right\rangle_{\mathcal{G}}$ obtained by discretizing the adjoint equations (optimize-then-discretize), and 'DFD' denote the fourth order finite difference approximation to the directional derivative $\left\langle\nabla\left(J_{h}(g)\right), \delta g\right\rangle_{\mathcal{G}}$ obtained 
by differentiating the discretized objective function.

Both axes of Fig. 10 have a logarithmic scale and the slope of each line measures the order of convergence as discretization is further refined. The observed rate of convergence for the objective function $J$ is approximately 5.5, while the relative errors between the directional derivative approximations converge with an observed rate of approximately 3. Since we use a finite difference scheme that is sixth order accurate in the interior and third order accurate near the boundary, since we apply a fourth order time stepping scheme, and since we employ sponge and Riemann extrapolation at the far field, it is difficult to conjecture what theoretical convergence rates should be obtained. However, our observed convergence rates for the various quantities are sensible. In particular, the comparison between the derivative information $\left\langle(\nabla J(g))_{h}, \delta g\right\rangle_{\mathcal{G}}$ obtained from our optimize-then-discretize approach and the finite difference approximation to the directional derivative $\left\langle\nabla\left(J_{h}(g)\right), \delta g\right\rangle_{\mathcal{G}}$ of the discretize-then-optimize approach support our procedure.

\section{Conclusions}

This work focuses on several important issues encountered in the optimal boundary control of aeroacoustic flows governed by high-order central finite difference discretizations of the unsteady Euler equations. These issues include: proper resolution of fluctuating flow quantities, nonreflecting farfield boundary treatments, and solid wall modeling. The importance of these issues is well known for computational aeroacoustics but they are also critically important when optimal control is applied to aeroacoustic flows. In addition, optimal transpiration boundary control of aeroacoustics also requires the formulation and implementation of accurate nearfield boundary conditions on the controlled segment of the boundary. It is argued, based on the characteristic wave propagation speeds normal to the controlled boundary, that subsonic suction requires one physical boundary condition while blowing requires three physical boundary conditions. Our implementation of the transpiration boundary condition is based on a decomposition of the inviscid fluxes into several planar pseudo-waves aligned with the boundary tangent and boundary normal direction using a technique originally introduced by Sesterhenn. ${ }^{1}$ The transpiration boundary condition is applied to three optimal control test problems. A continuous adjoint gradient-based method is used to solve the optimization problems and the adjoint equation, its end time condition, and boundary conditions are stated and discussed. Despite the difference in the number of derived adjoint boundary conditions for suction, blowing, and solid walls; the adjoint boundary conditions are compatible as the control tends to zero. The first test problem demonstrates that the transpiration control actuation is capable of producing well-resolved acoustic waves that reduce the observed sound amplitude by means of wave-cancellation. In the second test problem, the transpiration boundary condition mimics a nonreflecting boundary condition on the controlled surface in order to eliminate the intensifying effect of reflections off the solid wall. The final test problem demonstrates that 
optimal transpiration control is capable of creating a non-reflecting surface that rivals the widely used Riemann non-reflecting farfield treatment. The success of this approach has enabled us to investigate the feasibility of optimal transpiration control for reducing the impulsive noise associated with the Blade Vortex Interaction (BVI) phenomenon in Ref. 33.

\section{Appendix A: Adjoint Equations and Gradient of the Objective Function}

\section{Derivation of the adjoint equations}

We associate adjoint variables

$$
\boldsymbol{\lambda}=\left(\begin{array}{c}
\lambda_{1} \\
\lambda_{2} \\
\lambda_{3} \\
\lambda_{4}
\end{array}\right), \quad \boldsymbol{\lambda}^{b}=\left(\begin{array}{c}
\lambda_{1}^{b} \\
\lambda_{2}^{b} \\
\lambda_{3}^{b}
\end{array}\right), \quad \boldsymbol{\lambda}^{0}=\left(\begin{array}{c}
\lambda_{1}^{0} \\
\lambda_{2}^{0} \\
\lambda_{3}^{0} \\
\lambda_{4}^{0}
\end{array}\right)
$$

with the Euler equation (3a), its boundary conditions (3b) and initial conditions (3c), respectively. The Lagrangian corresponding to (3a)-(3c) and (7) is

$$
\begin{aligned}
L\left(\mathbf{u}, g, \boldsymbol{\lambda}, \boldsymbol{\lambda}^{b}, \boldsymbol{\lambda}^{0}\right)= & J(g)+\int_{t_{0}}^{t_{f}} \int_{\Omega} \boldsymbol{\lambda}^{T}\left(\mathbf{q}(\mathbf{u})_{t}+\sum_{i=1}^{2} \mathbf{F}^{i}(\mathbf{u})_{x_{i}}\right) d \mathbf{x} d t \\
& +\int_{t_{0}}^{t_{f}} \int_{\Gamma}\left(\boldsymbol{\lambda}^{b}\right)^{T} \mathbf{B}(\mathbf{u}, \nabla \mathbf{u}, g) d \mathbf{x} d t+\int_{\Gamma}\left(\boldsymbol{\lambda}^{0}\right)^{T}\left(\mathbf{u}-\mathbf{u}_{0}\right) d \mathbf{x} .
\end{aligned}
$$

The derivative $D J(g)$ of the objective function (7) applied to $g^{\prime}$ is given by

$$
\begin{aligned}
D J(g) g^{\prime}= & D_{g} L\left(\mathbf{u}, g, \boldsymbol{\lambda}, \boldsymbol{\lambda}^{b}, \boldsymbol{\lambda}^{0}\right) g^{\prime} \\
= & \int_{t_{0}}^{t_{f}} \int_{\Gamma_{c}}\left(\alpha_{1} g_{t} g_{t}^{\prime}+\alpha_{2} g g^{\prime}+\alpha_{3} \nabla g \nabla g^{\prime}+\alpha_{4} \Delta g \Delta g^{\prime}\right) d \mathbf{x} d t \\
& +\int_{t_{0}}^{t_{f}} \int_{\Gamma}\left(\boldsymbol{\lambda}^{b}\right)^{T} \mathbf{B}_{g}(\mathbf{u}, \nabla \mathbf{u}, g) g^{\prime} d \mathbf{x} d t
\end{aligned}
$$

where $\boldsymbol{\lambda}, \boldsymbol{\lambda}^{b}, \boldsymbol{\lambda}^{0}$ are the solution of the adjoint equations $D_{\mathbf{u}} L\left(\mathbf{u}, g, \boldsymbol{\lambda}, \boldsymbol{\lambda}^{b}, \boldsymbol{\lambda}^{0}\right) \mathbf{u}^{\prime}=\mathbf{0}$ for all $\mathbf{u}^{\prime}$, i.e.

$$
\begin{aligned}
& \int_{t_{0}}^{t_{f}} \int_{\Omega}\left(\mathbf{u}^{\prime}\right)^{T} \mathbf{r}+\int_{t_{0}}^{t_{f}} \int_{\Omega}(\boldsymbol{\lambda})^{T}\left(\left(\mathbf{M} \mathbf{u}^{\prime}\right)_{t}+\sum_{i}\left(\mathbf{A}^{i} \mathbf{u}^{\prime}\right)_{x_{i}}\right) \\
& \quad+\int_{t_{0}}^{t_{f}} \int_{\Gamma}\left(\boldsymbol{\lambda}^{b}\right)^{T}\left(\mathbf{B}_{\mathbf{u}} \mathbf{u}^{\prime}+\sum_{i} \mathbf{B}_{\mathbf{u}_{x_{i}}} \mathbf{u}_{x_{i}}^{\prime}\right)+\int_{\Omega}\left(\mathbf{u}^{\prime}\right)^{T} \boldsymbol{\lambda}^{0}=\mathbf{0} \quad \forall \mathbf{u}^{\prime} .
\end{aligned}
$$

In (25) $\mathbf{r}$ is defined by (9). Integration by parts in (25) and varying over all functions $\mathbf{u}^{\prime}$ leads to 
the equations

$$
\begin{array}{rr}
\mathbf{M}^{T} \boldsymbol{\lambda}_{t}+\sum_{i} \mathbf{A}^{i^{T}} \boldsymbol{\lambda}_{x_{i}}^{d}=\mathbf{r} & \left(t_{0}, t_{f}\right) \times \Omega, \\
\boldsymbol{\lambda}=\mathbf{0} & \left\{t_{f}\right\} \times \Omega, \\
\boldsymbol{\lambda}^{0}-\left.\left(\mathbf{M}^{T} \boldsymbol{\lambda}\right)\right|_{t=t_{0}}=\mathbf{0} & \left\{t_{0}\right\} \times \Omega, \\
\sum_{j} n_{j} \mathbf{B}_{\mathbf{u}_{x_{j}}^{T}}^{T} \boldsymbol{\lambda}^{b}=\mathbf{0} & \left(t_{0}, t_{f}\right) \times \Gamma, \\
\sum_{i} n_{i} \mathbf{A}^{i T} \boldsymbol{\lambda}^{d}+\mathbf{B}_{\mathbf{u}}^{T} \boldsymbol{\lambda}^{b}-\partial_{s} \sum_{j} s_{j} \mathbf{B}_{\mathbf{u}_{x_{j}}}^{T} \boldsymbol{\lambda}^{b}=\mathbf{0} & \left(t_{0}, t_{f}\right) \times \Gamma,
\end{array}
$$

where, as before, $\mathbf{s}=\left(s_{1}, s_{2}\right)^{T}=\left(-n_{2}, n_{1}\right)^{T}$ is the unit tangential vector. For additional details on the derivation of (26a)-(26e) see Ref 3.

Equation (26a) gives the adjoint partial differential equation (PDE) for $\lambda$, (26b) specifies the final time condition for the adjoint PDE and (26d), (26e) determine the boundary conditions for $\lambda$. The equations (26d) and (26e) also determine the relation between the adjoint variables $\boldsymbol{\lambda}$ and $\boldsymbol{\lambda}^{b}$. Recall that $\lambda^{b}$ is needed for the computation of the derivative (24) of the objective functional. In fact, since

$$
\left(\boldsymbol{\lambda}^{b}\right)^{T} \mathbf{B}_{g}(\mathbf{u}, \nabla \mathbf{u}, g)=-\lambda_{1}^{b}
$$

$\lambda_{2}^{b}$ and $\lambda_{3}^{b}$ are not needed. In the following we derive the adjoint boundary conditions for $\lambda$ determined by (26d) and (26e) as well as the relation between $\lambda$ and $\lambda_{1}^{b}$ specified by the same equations.

Equations (11)-(13) state the adjoint boundary conditions in terms of the adjoint variables $\lambda$. For the derivation of these conditions and for their implementation it is convenient to introduce the characteristic adjoint variables $\Psi$, which will be defined below. By $\mathbf{M}=\partial \mathbf{q} / \partial \mathbf{u}$ we denote the Jacobian of the conservative variables with respect to the primitive variables. We recall that $\mathbf{A}^{i}=\partial \mathbf{F}^{i} / \partial \mathbf{u}$. Hence $\mathbf{A}^{i}=\partial \mathbf{F}^{i} / \partial \mathbf{q} \mathbf{M}$. It is well known (see, e.g., $\S 16.5 .2$ in Ref. 29) that

$$
\sum_{i} n_{i}\left(\frac{\partial \mathbf{F}^{i}}{\partial \mathbf{q}}\right)=\mathbf{P} \boldsymbol{\Lambda} \mathbf{P}^{-1}
$$

where $\Lambda$ is a diagonal matrix with diagonal entries $\mathbf{v}^{T} \mathbf{n}, \mathbf{v}^{T} \mathbf{n}, \mathbf{v}^{T} \mathbf{n}+c, \mathbf{v}^{T} \mathbf{n}-c$, and

$$
\mathbf{P}=\left(\begin{array}{cccc}
1 & 0 & \frac{\rho}{2 c} & \frac{\rho}{2 c} \\
v_{1} & \rho n_{2} & \frac{\rho}{2 c}\left(v_{1}+c n_{1}\right) & \frac{\rho}{2 c}\left(v_{1}-c n_{1}\right) \\
v_{2} & -\rho n_{1} & \frac{\rho}{2 c}\left(v_{2}+c n_{2}\right) & \frac{\rho}{2 c}\left(v_{2}-c n_{2}\right) \\
\frac{\mathbf{v}^{T} \mathbf{v}}{2} & \rho\left(v_{1} n_{2}-v_{2} n_{1}\right) & \frac{\rho}{2 c}\left(H+c \mathbf{v}^{T} \mathbf{n}\right) & \frac{\rho}{2 c}\left(H-c \mathbf{v}^{T} \mathbf{n}\right)
\end{array}\right)
$$

Here $c=\sqrt{\gamma p / \rho}$ denotes the speed of sound and $H=\frac{1}{2} \mathbf{v}^{T} \mathbf{v}+c^{2} /(\gamma-1)$ is the stagnation 
enthalpy. With

$$
\mathbf{L}^{-1}=\mathbf{P}^{-1} \mathbf{M}=\left(\begin{array}{cccc}
\frac{\gamma-1}{\gamma} & 0 & 0 & \frac{-\rho}{\gamma T} \\
0 & n_{2} & -n_{1} & 0 \\
\frac{c}{\gamma p} & n_{1} & n_{2} & \frac{c}{\gamma T} \\
\frac{c}{\gamma p} & -n_{1} & -n_{2} & \frac{c}{\gamma T}
\end{array}\right)
$$

we obtain

$$
\sum_{i} n_{i} \mathbf{A}^{i^{T}}=\mathbf{M}^{T} \sum_{i} n_{i}\left(\frac{\partial \mathbf{F}^{i}}{\partial \mathbf{q}}\right)^{T}=\mathbf{M}^{T}\left(\mathbf{P} \boldsymbol{\Lambda} \mathbf{P}^{-1}\right)^{T}=\mathbf{L}^{-T} \boldsymbol{\Lambda} \mathbf{P}^{T}
$$

This identity together with the definition

$$
\Psi=\mathbf{P}^{T} \boldsymbol{\lambda}
$$

of the characteristic adjoint variables will be used to rewrite (26e). Refs. 15, 34-36 have also used adjoint characteristic variables in the formulation and implementation of adjoint boundary conditions.

\section{Adjoint wall boundary conditions}

Because the number of imposed boundary conditions and the imposed quantities themselves depend on the sign of the normal velocity control, we discuss suction and blowing boundary conditions separately.

Suction $\left(\mathbf{v}^{T} \mathbf{n}=g>0\right)$

The suction boundary condition operator and its derivatives are given by

$$
\mathbf{B}(\mathbf{u}, \nabla \mathbf{u}, g)=\left(\begin{array}{c}
n_{1} v_{1}+n_{2} v_{2}-g \\
0 \\
0
\end{array}\right), \quad \mathbf{B}_{\mathbf{u}}=\left(\begin{array}{cccc}
0 & n_{1} & n_{2} & 0 \\
0 & 0 & 0 & 0 \\
0 & 0 & 0 & 0
\end{array}\right), \quad \mathbf{B}_{\mathbf{u}_{x_{j}}}=\mathbf{0}
$$

Using (30) and (31) the equations (26e) are given by

$$
\left(\begin{array}{cccc}
\frac{\gamma-1}{\gamma} & 0 & \frac{c}{\gamma p} & \frac{c}{\gamma p} \\
0 & n_{2} & n_{1} & -n_{1} \\
0 & -n_{1} & n_{2} & -n_{2} \\
\frac{-\rho}{\gamma T} & 0 & \frac{c}{\gamma T} & \frac{c}{\gamma T}
\end{array}\right)\left(\begin{array}{c}
\mathbf{v}^{T} \mathbf{n} \psi_{1} \\
\mathbf{v}^{T} \mathbf{n} \psi_{2} \\
\left(\mathbf{v}^{T} \mathbf{n}+c\right) \psi_{3} \\
\left(\mathbf{v}^{T} \mathbf{n}-c\right) \psi_{4}
\end{array}\right)+\left(\begin{array}{c}
0 \\
n_{1} \lambda_{1}^{b} \\
n_{2} \lambda_{1}^{b} \\
0
\end{array}\right)=\mathbf{0} .
$$

Multiplying the first equation in (32) by $p$ and subtracting from the result $T$ times the fourth equation leads to

$$
\left(\mathbf{v}^{T} \mathbf{n}\right) \psi_{1}=0
$$


The fourth equation of (32) and (33) give the second adjoint boundary condition

$$
\left(\mathbf{v}^{T} \mathbf{n}+c\right) \psi_{3}+\left(\mathbf{v}^{T} \mathbf{n}-c\right) \psi_{4}=0
$$

If we multiply the second and third components of (32) by $n_{2}$ and $-n_{1}$, respectively, and add the results, we obtain

$$
\left(\mathbf{v}^{T} \mathbf{n}\right) \psi_{2}=0
$$

Equations (33)-(35) are the adjoint boundary conditions (11) stated earlier.

If we multiply the second component of (32) by $n_{1}$ and the third component by $n_{2}$ and add the results, we obtain

$$
\lambda_{1}^{b}=\left(\mathbf{v}^{T} \mathbf{n}-c\right) \psi_{4}-\left(\mathbf{v}^{T} \mathbf{n}+c\right) \psi_{3}
$$

The adjoint variable $\lambda_{1}^{b}$ is the only component of $\boldsymbol{\lambda}^{b}$ needed for the computation of the objective function derivative (see (24), (27)).

Solid surface $\left(\mathbf{v}^{T} \mathbf{n}=g=0\right)$

On a uncontrolled solid surface equations (33) and (35) are identically satisfied and only (34) needs to be imposed.

Blowing $\left(\mathbf{v}^{T} \mathbf{n}=g<0\right)$

In the case $\mathbf{v}^{T} \mathbf{n}=g<0$, the boundary condition operator and its derivatives are given by

$$
\mathbf{B}(\mathbf{u}, \nabla \mathbf{u}, g)=\left(\begin{array}{c}
n_{1} v_{1}+n_{2} v_{2}-g \\
\left(S-S_{0}\right) g^{2} \\
\left(\partial_{x_{2}} v_{1}-\partial_{x_{1}} v_{2}\right) g^{2}
\end{array}\right)
$$

and

$$
\mathbf{B}_{\mathbf{u}}=\left(\begin{array}{cccc}
0 & n_{1} & n_{2} & 0 \\
\frac{-g^{2}}{\rho} & 0 & 0 & \frac{g^{2}}{(\gamma-1) T} \\
0 & 0 & 0 & 0
\end{array}\right), \quad \mathbf{B}_{\mathbf{u}_{x_{j}}}=\left(\begin{array}{cccc}
0 & 0 & 0 & 0 \\
0 & 0 & 0 & 0 \\
0 & \delta_{2 j} g^{2} & -\delta_{1 j} g^{2} & 0
\end{array}\right)
$$

where $\delta_{i j}$ denotes the Kronecker symbol. Equation (26d) implies

$$
\lambda_{3}^{b}=0
$$


Equations (37), (30), and (31) can be used to write equation (26e) as

$$
\left(\begin{array}{cccc}
\frac{\gamma-1}{\gamma} & 0 & \frac{c}{\gamma p} & \frac{c}{\gamma p} \\
0 & n_{2} & n_{1} & -n_{1} \\
0 & -n_{1} & n_{2} & -n_{2} \\
\frac{-\rho}{\gamma T} & 0 & \frac{c}{\gamma T} & \frac{c}{\gamma T}
\end{array}\right)\left(\begin{array}{c}
\mathbf{v}^{T} \mathbf{n} \psi_{1} \\
\mathbf{v}^{T} \mathbf{n} \psi_{2} \\
\left(\mathbf{v}^{T} \mathbf{n}+c\right) \psi_{3} \\
\left(\mathbf{v}^{T} \mathbf{n}-c\right) \psi_{4}
\end{array}\right)+\left(\begin{array}{c}
-\frac{g^{2}}{\rho} \lambda_{2}^{b} \\
n_{1} \lambda_{1}^{b} \\
n_{2} \lambda_{1}^{b} \\
\frac{g^{2}}{(\gamma-1) T} \lambda_{2}^{b}
\end{array}\right)=\mathbf{0}
$$

Notice that the second and third components in (38) are identical to the second and third components in (32). Hence we obtain (35) and (36).

Another boundary condition for $\lambda$ is obtained from (38). We can eliminate $\lambda_{2}^{b}$ from the first and fourth components of (38) by multiplying these components by $\rho$ and $(\gamma-1) T$ respectively, and adding the results to obtain

$$
\left(\mathbf{v}^{T} \mathbf{n}+c\right) \psi_{3}+\left(\mathbf{v}^{T} \mathbf{n}-c\right) \psi_{4}=0 .
$$

In summary, there are two boundary conditions that need to be imposed on $\psi$, namely, (39) and (35). These boundary conditions are equivalent to (13). As in the previous case, the adjoint variable $\lambda_{1}^{b}$ needed for the computation of the objective function derivative (see (24), (27)) is obtained from (36). It is interesting to note that the vorticity boundary condition of the state only leads to the condition (37). Because it involves only derivatives of the primitive variables, this boundary condition does not enter (38). A consequence of this is the appearance of the additional boundary condition (35).

\section{Gradient computation}

Given $\lambda_{1}^{b}$, the gradient of the objective function can now be computed from (24). Details may be found in Ref. 2.

\section{Acknowledgments}

This work was supported in part by Texas ATP grant 003604-0001-1999, NSF grant DMS0075731, and Los Alamos National Laboratory Computer Science Institute (LACSI) through LANL contract number 03891-99-23 as part of the prime contract (W-7405-ENG-36) between the Department of Energy and the Regents of the University of California. Computations were performed on an SGI Origin 2000 which was purchased with the aid of NSF SCREMS grant 9872009. 


\section{References}

${ }^{1}$ Sesterhenn, J., "A Characteristic-Type Formulation of the Navier-Stokes Equations for High Order Upwind Schemes," Computers \& Fluids, Vol. 30, 2001, pp. 37-67.

${ }^{2}$ Collis, S. S., Ghayour, K., Heinkenschloss, M., Ulbrich, M., and Ulbrich, S., "Optimal Control of Unsteady Compressible Viscous Flows," Inter. J. Num. Meth. Fluids, Vol. 40, 2002, pp. 1401-1429.

${ }^{3}$ Collis, S. S., Ghayour, K., Heinkenschloss, M., Ulbrich, M., and Ulbrich, S., "Numerical Solution of Optimal Control Problems by the Compressible Navier-Stokes Equations," Proceedings of the International Conference on Optimal Control of Complex Structures, edited by G. Leugering, J. Sprekels, and F. Tröltzsch, Vol. 139, Birkhäuser Verlag, 2001, pp. 43-55.

${ }^{4}$ Tam, C. K. W. and Dong, Z., "Wall Boundary Conditions for High-Order Finite Difference Schemes in Computational Aeroacoustics," Theoretical and Computational Fluid Dynamics, Vol. 6, No. 6, 1994, pp. 303-322.

${ }^{5}$ Kurbatskii, K. A. and Tam, C. K. W., "Cartesian Boundary Treatment of Curved Walls for High-Order Computational Aeroacoustics Schemes,” AIAA J., Vol. 35, No. 1, 1997, pp. 133-140.

${ }^{6}$ Joslin, R. D., Gunzburger, M. D., Nicolaides, R. A., Erlebacher, G., and Hussaini, M. Y., "Self-Contained Automated Methodology for Optimal Flow Control," AIAA J., Vol. 35, No. 5, 1997, pp. 816-824.

${ }^{7}$ Abergel, F. and Temam, R., "On some Control Problems in Fluid Mechanics," Theoretical and Computational Fluid Dynamics, Vol. 1, 1990, pp. 303-325.

${ }^{8}$ Berggren, M., "Numerical Solution of a Flow-Control Problem: Vorticity Reduction by Dynamic Boundary Action," SIAM J. Scientific Computing, Vol. 19, 1998, pp. 829-860.

${ }^{9}$ He, J.-W., Glowinski, R., Metcalfe, R., Nordlander, A., and Periaux, J., "Active Control and Drag Optimization for Flow past a Circular Cylinder. I. Oscillatory Cylinder Rotation," Journal of Computational Physics, Vol. 163, 2000, pp. 83-117.

${ }^{10}$ Fursikov, A. V., Gunzburger, M. D., and Hou, L. S., "Boundary value Problems and Optimal Boundary Control for the Navier-Stokes Systems: The Two-Dimensional Case," SIAM J. Control Optimization, Vol. 36, 1998, pp. 852-894.

${ }^{11}$ Gunzburger, M. D. and Manservisi, S., "The velocity tracking problem for Navier-Stokes flows with boundary control," SIAM J. Control Optimization, Vol. 39, 2000, pp. 594-634.

${ }^{12} \mathrm{He}$, B., Ghattas, O., and Antaki, J. F., "Computational Strategies for Shape Optimization of Time-Dependent Navier-Stokes Flows,” Tech. Rep. CMU-CML-97-102, Carnegie Mellon University, June 1997. 
${ }^{13}$ Li, Z., Navon, M., Hussaini, M. Y., and Dimet, F. X. L., “Optimal Control of Cylinder Wakes via Suction and Blowing," Computers and Fluids, Vol. 32, No. 2, 2003, 149-171.

${ }^{14}$ Anderson, W. K. and Venkatakrishnan, V., "Aerodynamic Design Optimization on Unstructured Grids with a Continuous Adjoint Formulation,” Tech. Rep. 97-9, ICASE, NASA Langley Research Center, Hampton VA 23681-0001, 1997.

${ }^{15}$ Giles, M. B. and Pierce, N. A., "Adjoint equations in CFD: Duality, boundary conditions and solution behavior," AIAA Paper 97-1850, 1997, Available electronically from URL http://www.comlab.ox.ac.uk/oucl/users/mike.giles/conferences.html.

${ }^{16}$ Iollo, A. and Salas, M. D., "Optimum Transonic Airfoils Based on the Euler Equations," Tech. Rep. 96-76, ICASE, NASA Langley Research Center, Hampton VA 23681-0001, 1996, Available electronically from URL ftp://ftp.icase.edu/pub/techreports/96/96-76.ps.

${ }^{17}$ Jameson, A. and Reuther, J., "Control Theory Based Airfoil Design Using the Euler Equations," AIAA Paper 94-4272, 1994.

${ }^{18}$ Nadarajah, S. K. and Jameson, A., "A Comparison of the Continuous and Discrete Adjoint Approach to Automatic Aerodynamic Optimization," AIAA Paper 2000-0667, 2000.

${ }^{19}$ Lele, S. K., "Compact finite difference schemes with spectral-like resolution," J. Comp. Phys., Vol. 103, No. 1, 1992, pp. 16-42.

${ }^{20}$ Tam, C. K. W. and Webb, J. C., "Dispersion-Relation-Preserving Finite Difference Schemes for Computational Acoustics," J. Comp. Phys., Vol. 107, 1993, pp. 262-281.

${ }^{21}$ Collis, S. S., A Computational Investigation of Receptivity in High-Speed Flow Near a Swept Leading-Edge, Ph.D. thesis, Stanford University, March 1997, available electronically from http://mems.rice.edu/ collis/papers/.

${ }^{22}$ Carpenter, M. H., Gottlieb, D., and Abarbanel, S., "Stable and Accurate Boundary Treatments for Compact, High-Order Finite Difference Schemes," Appl. Num. Math., Vol. 12, No. 1-3, 1993, pp. 55-87.

${ }^{23}$ Israeli, M. and Orszag, S. A., “Approximation of Radiation Boundary Condition,” Journal of Computational Physics, Vol. 41, 1981, pp. 115-135.

${ }^{24}$ Thompson, K. W., "Time-Dependent Boundary Conditions for Hyperbolic Systems," J. Comp. Phys., Vol. 68, 1987, pp. 1-24.

${ }^{25}$ Thompson, K. W., “Time-Dependent Boundary Conditions for Hyperbolic Systems, II,” J. Comp. Phys., Vol. 89, 1990, pp. 439-461.

${ }^{26}$ Poinsot, T. J. and Lele, S. K., "Boundary Conditions for Direct Simulations of Compressible Viscous Flows," J. Comp. Phys., Vol. 101, No. 1, 1992, pp. 104-129. 
${ }^{27}$ Giles, M. B., "Nonreflecting Boundary Conditions for Euler Equation Calculations," AIAA J., Vol. 28, No. 12, 1990, pp. 2050-2058.

${ }^{28}$ Moretti, G., “The $\lambda$-scheme," Computers \& Fluids, Vol. 7, 1979, pp. 191-205.

${ }^{29}$ Hirsch, C., Numerical Computation of Internal and External Flows, Vol. 2, John Wiley \& Sons, 1990.

${ }^{30}$ Shu, C.-W., "Esentially Non-Oscillatory and Weighted Essentially Non-Oscillatory Schemes for Hyperbolic Conservation Laws," Tech. Rep. 97-65, ICASE, November 1997.

${ }^{31}$ Hager, W. W., "Runge-Kutta methods in optimal control and the transformed adjoint system," Numerische Mathematik, Vol. 87, 2000, pp. 247-282.

${ }^{32}$ Vogel, C. R. and Wade, J. G., "Analysis of Costate Discretizations in Parameter Estimation for Linear Evolution Equations," SIAM J. Control and Optimization, Vol. 33, 1995, pp. 227-254.

${ }^{33}$ Collis, S. S., Ghayour, K., and Heinkenschloss, M., "Optimal Control of Aeroacoustic Noise Generated by Cylinder Vortex Interaction," International Journal of Aeroacoustics, Vol. 1, 2002, pp. 97-114.

${ }^{34}$ Sanders, B. F. and Katopodes, N. D., "Control of canal flow by adjoint sensitivity," Journal of Irrigation abd Drainage Engineering E-ASCE, Vol. 125, No. 5, 1999, pp. 287-297.

${ }^{35}$ Sanders, B. F. and Katopodes, N. D., “Adjoint Sensitivity Analysis for Shallow-Water Wave Control," Journal of Engineering Mechanics-ASCE, Vol. 126, No. 9, 2000, pp. 909-919.

${ }^{36}$ Baysal, O. and Ghayour, K., "Continuous Adjoint Sensitivities for General Cost Functionals on Unstructured Meshes in Aerodynamic Shape Optimization," AIAA J., Vol. 39, No. 1, 2001, pp. $48-55$. 


\section{List of Figure Captions}

Figure 1: Pseudo-waves at a subsonic boundary point.

Figure 2: A schematic diagram of test case 1

Figure 3: Contours of $p-p_{\mathrm{a}}$ at four equally spaced instants spanning one period of oscillation $T_{p}$ ( 6 equally spaced contour levels between $\left.\pm 7.0 \times 10^{-6}\right)$

Figure 4: Time history of control, $g$.

a): $x=0$.

b): $x=0, \pm 1 / 4$.

Figure 5: Effect of control for case 1.

a): Control $g$ near the final time $t_{f}$

b): $p_{\text {optimal }}-p_{\text {nocontrol }}$ at time $t=40.5$. (20 evenly spaced contours between $\pm 3.5 \times 10^{-6}$ )

Figure 6: Contours of $p-p_{\mathrm{a}}$ at four equally spaced instants spanning one period of motion $T_{p}$ ( 6 contour levels between $\left.\pm 1.75 \times 10^{-4}\right)$

Figure 7: Effect of control for case 2.

a): Time history of control $g$ at $x_{1}=0, \pm 1 / 4$

b): $p_{\text {optimal }}-p_{\text {nocontrol }}$ at time $40.75\left(20\right.$ contours between $\pm 9.0 \times 10^{-5}$ )

Figure 8: Contours of $p-p_{\mathrm{a}}$ at instants $t=2,4,8,9$ for no control (left) and optimal control (right). (41 contour levels between $8.12 \times 10^{-6}$ and $4.74 \times 10^{-4}$ )

Figure 9: Time history of pressure fluctuations at $(0,7)$ for case 3 .

Figure 10: Mesh refinement study for test case 3. 


\section{Interior Domain}

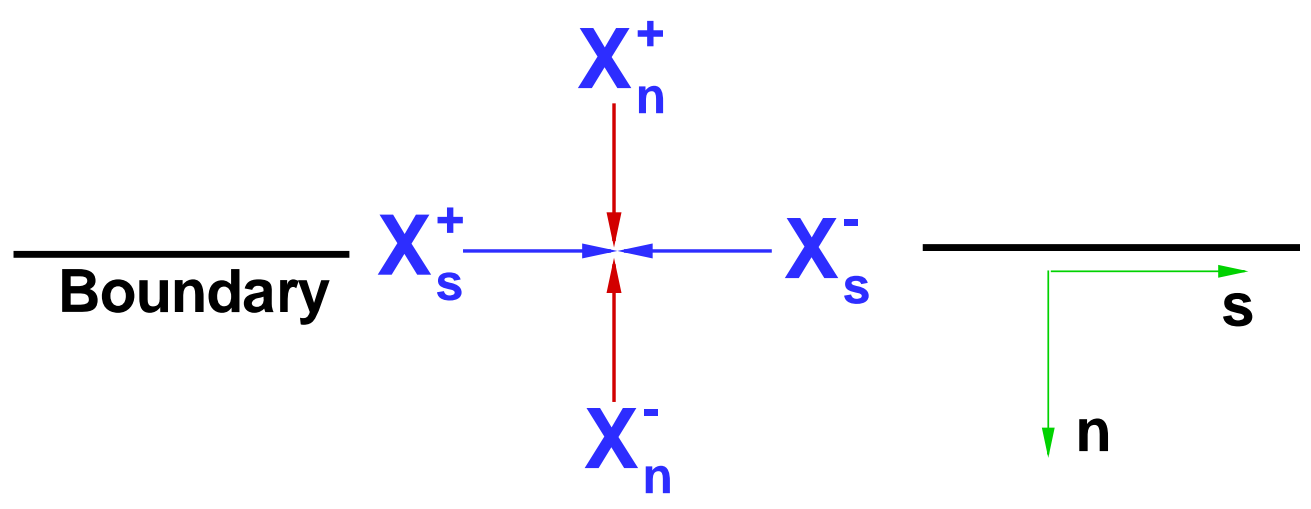




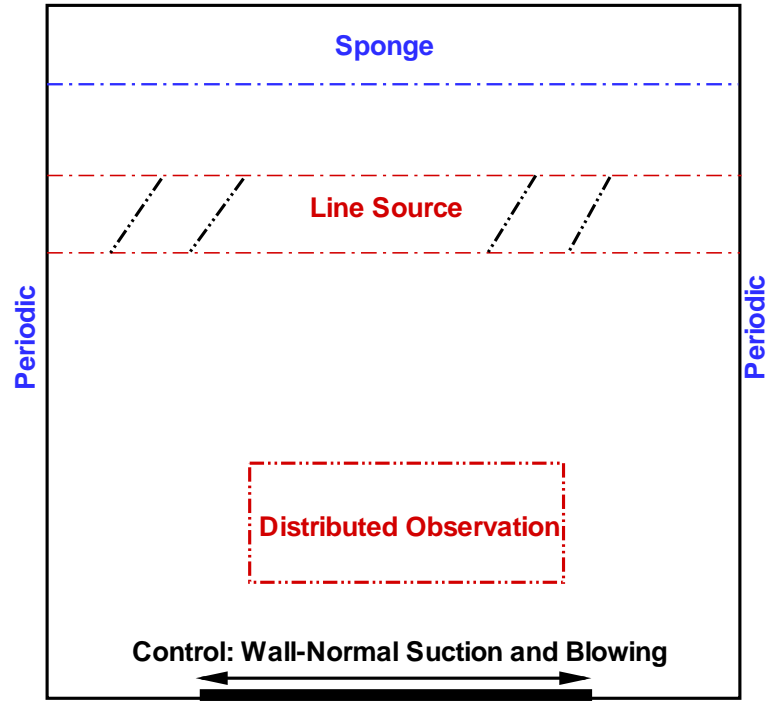




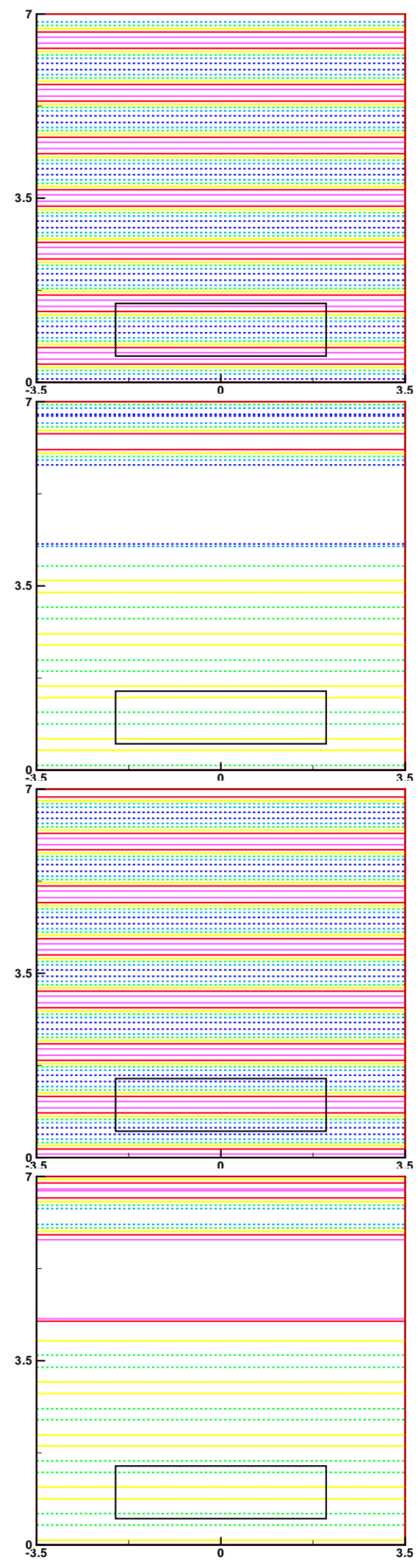

(a) No control

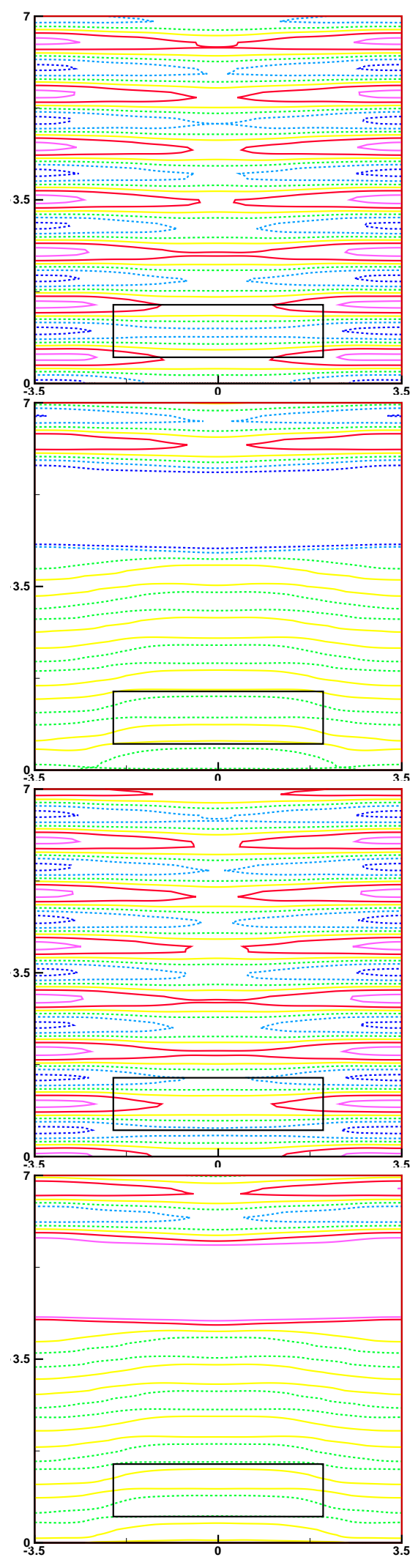

(b) Optimal control 

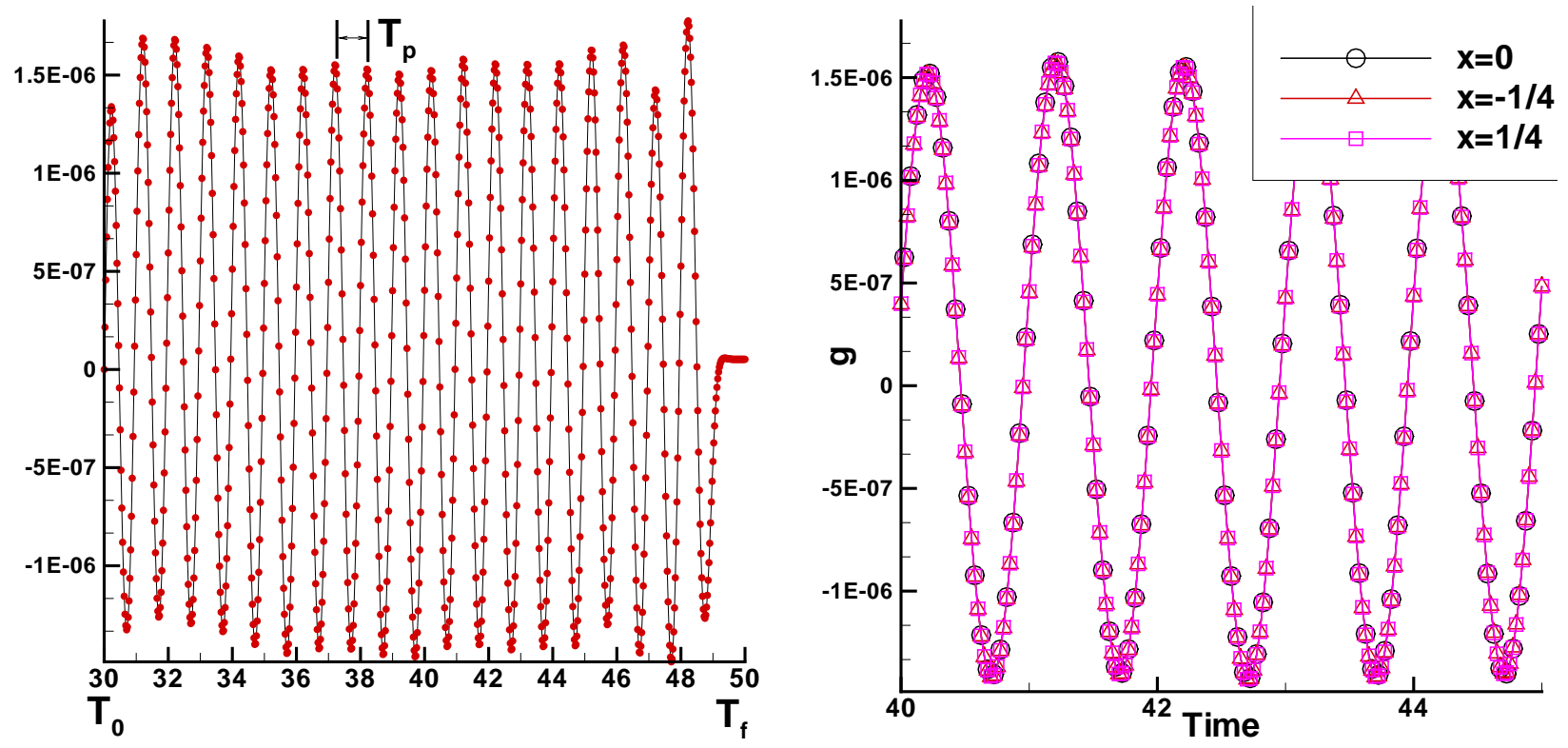

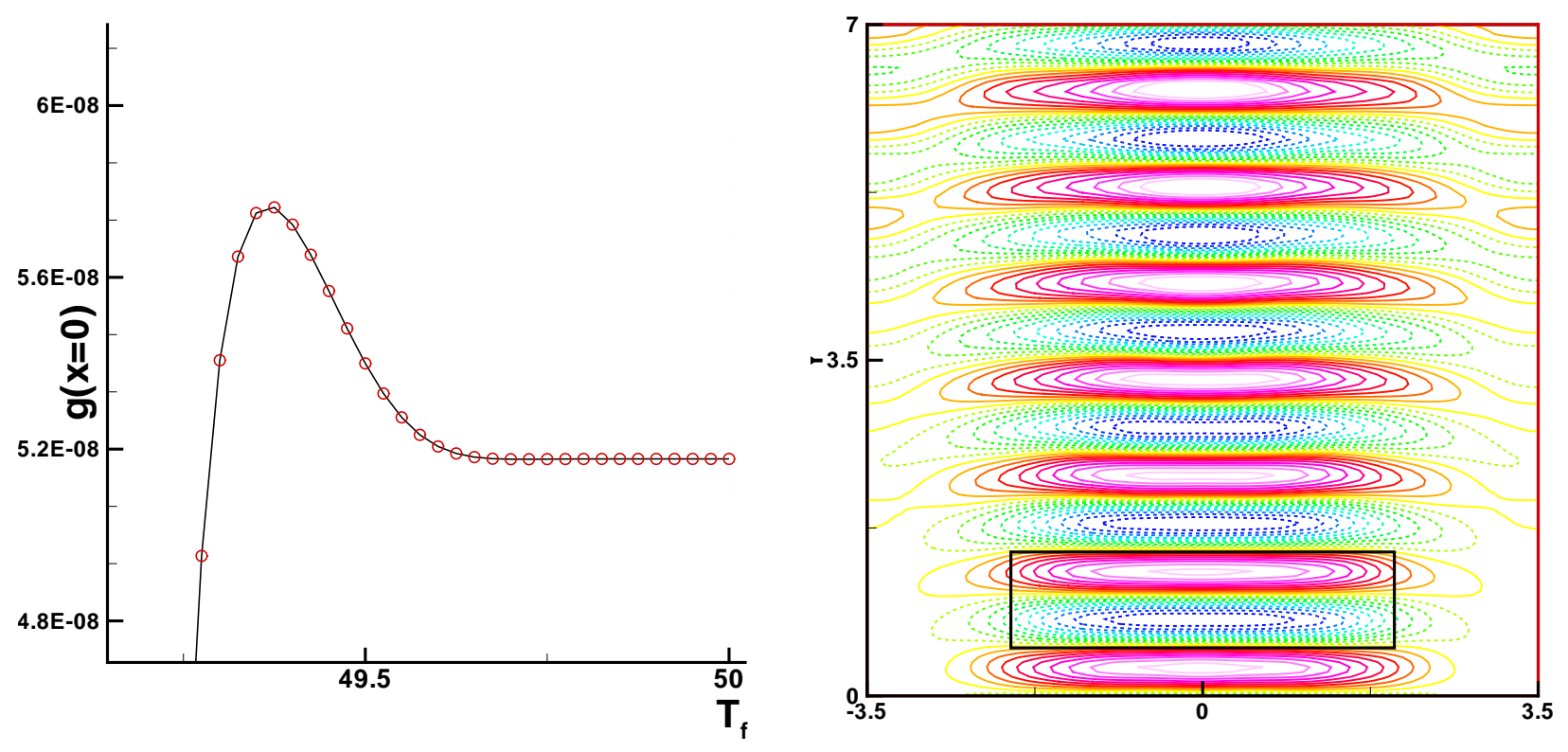


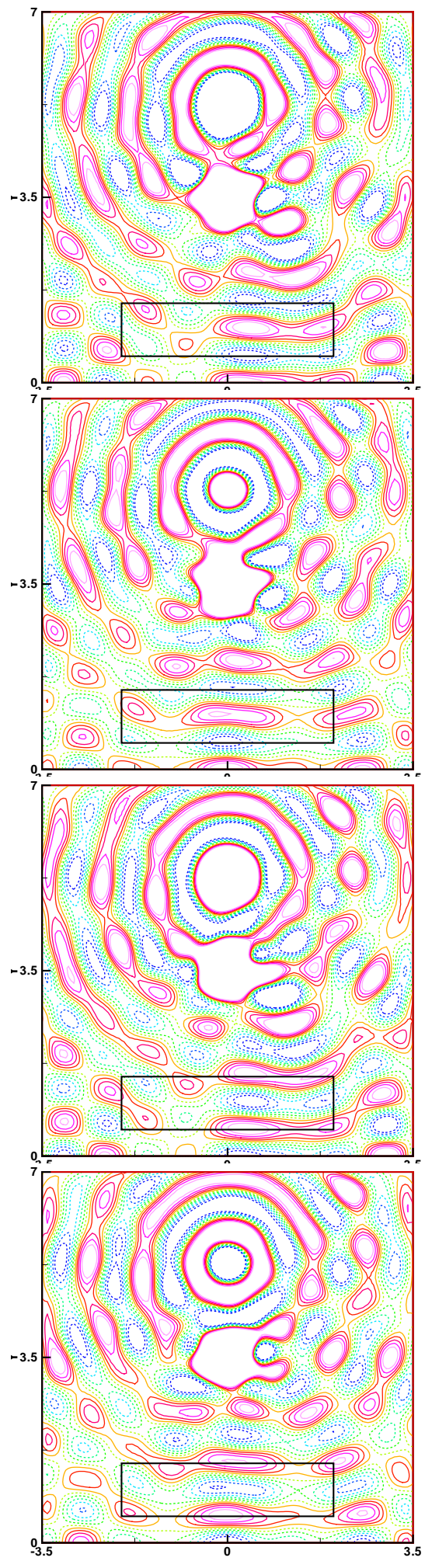

(a) No control

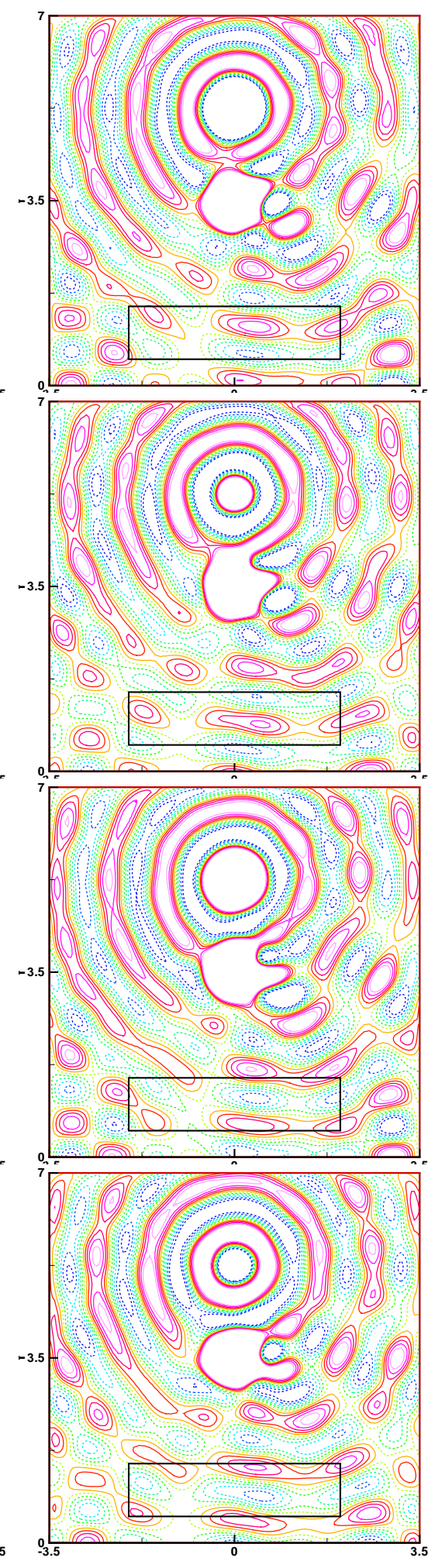

(b) Optimal control

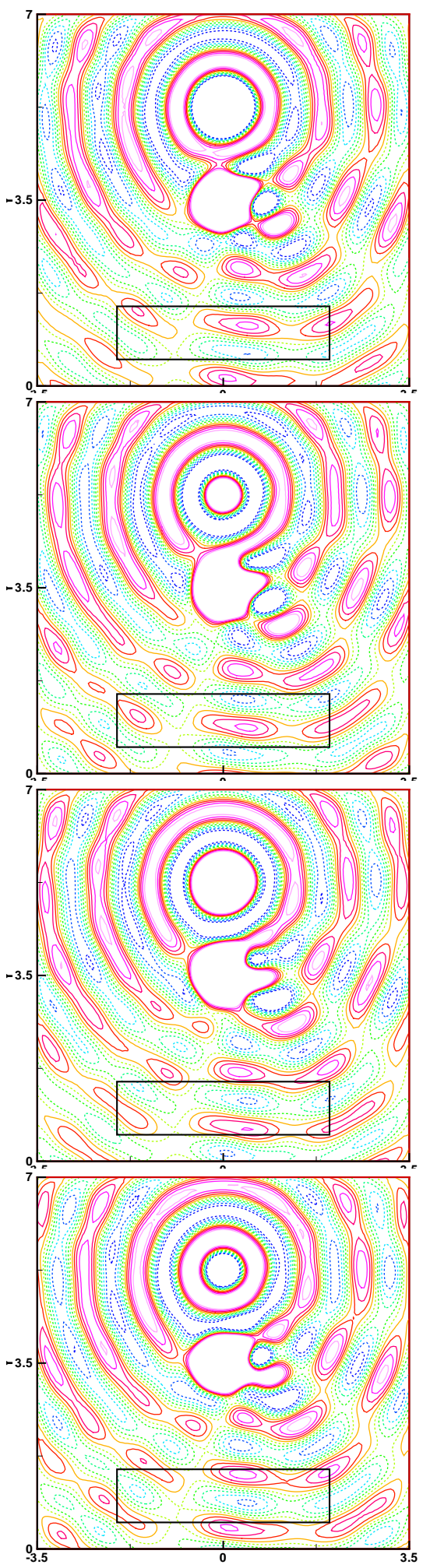

(c) Far-field BC on bottom boundary 

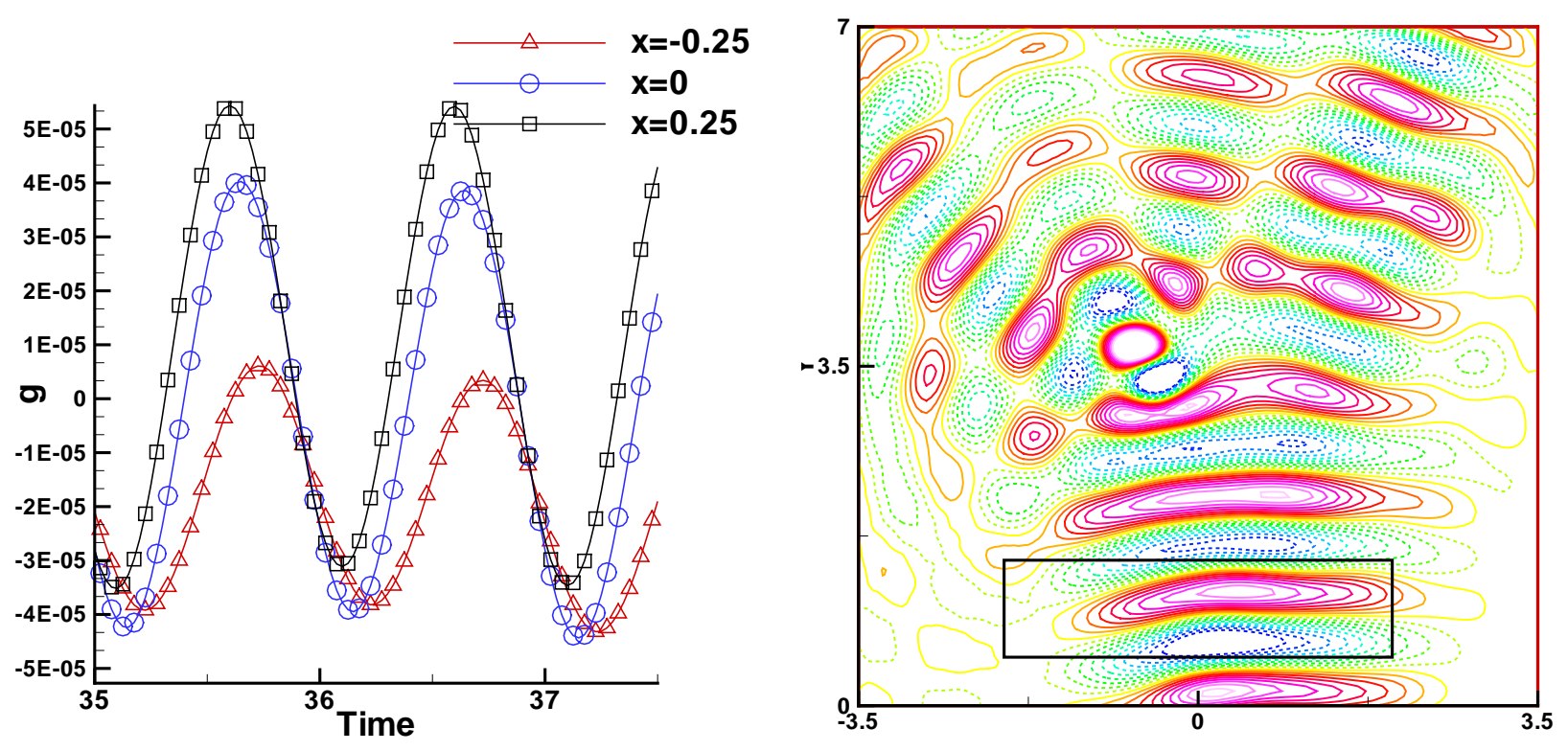

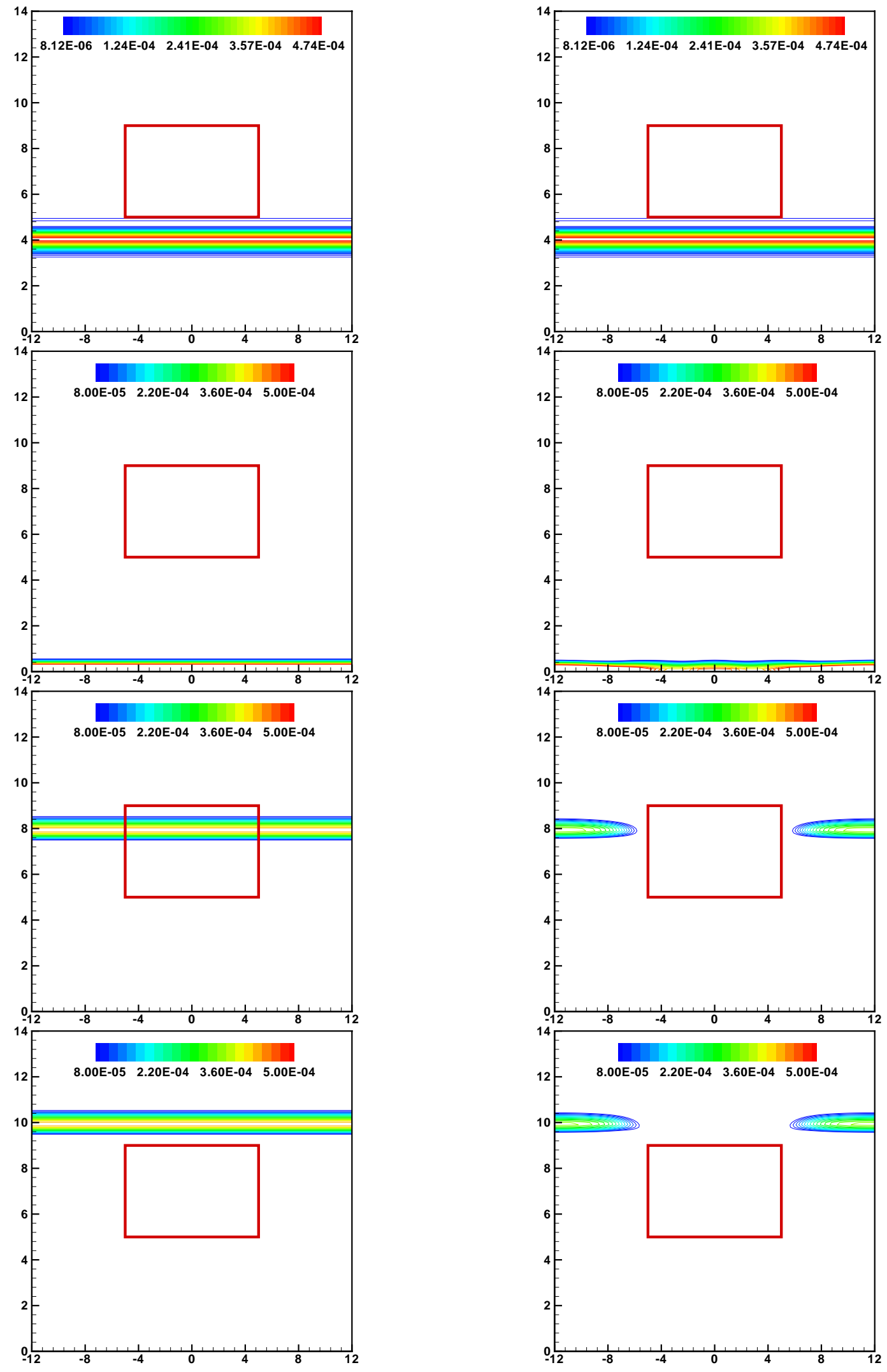

(a) No control

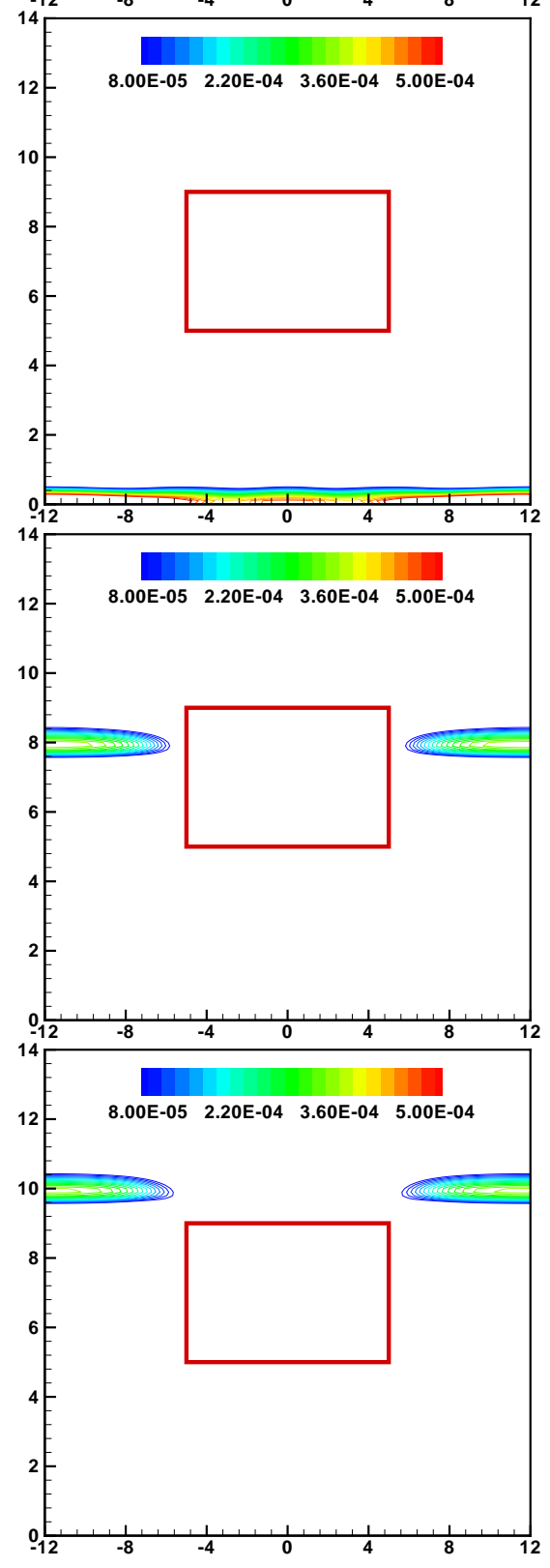

(b) Optimal control 


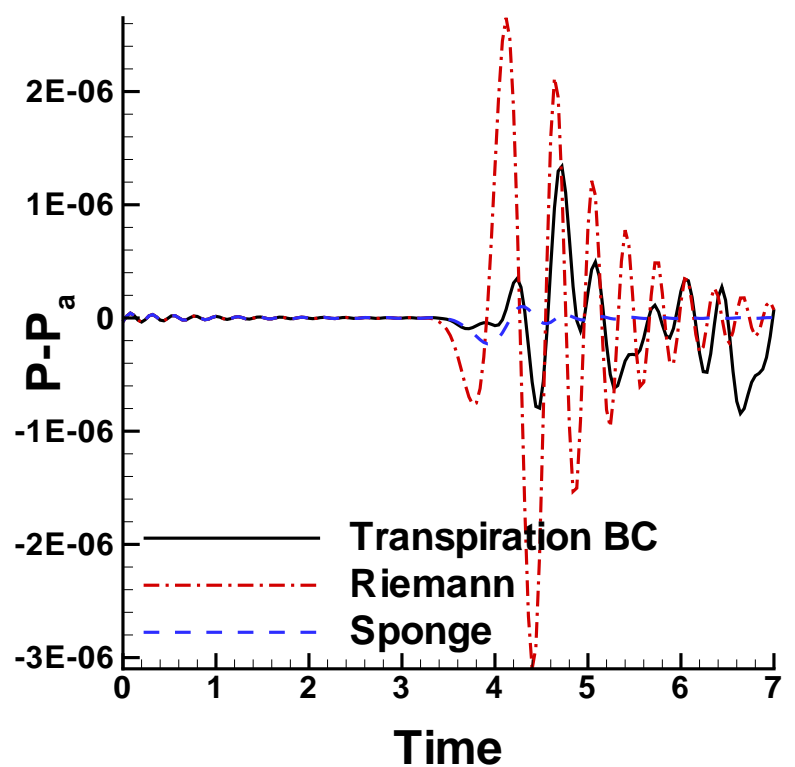




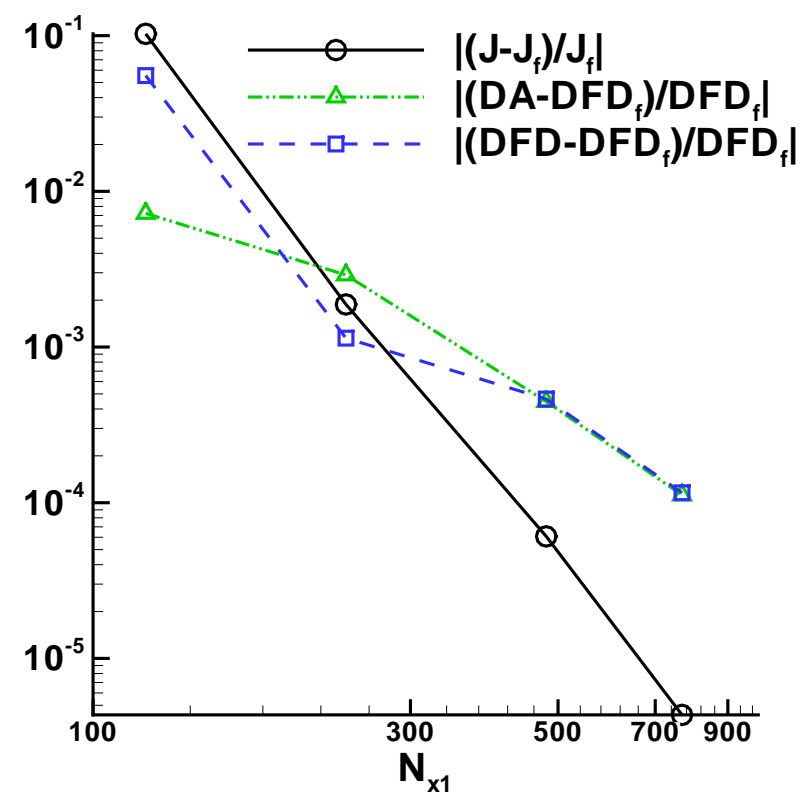

